\title{
A VARIATIONAL APPROXIMATION SCHEME FOR RADIAL POLYCONVEX ELASTICITY THAT PRESERVES THE POSITIVITY OF JACOBIANS*
}

\author{
ALEXEY MIROSHNIKOV ${ }^{\dagger}$ AND ATHANASIOS E. TZAVARAS $\ddagger$
}

To Dave Levermore on his 60th birthday with friendship and appreciation

\begin{abstract}
We consider the equations describing the dynamics of radial motions for isotropic elastic materials; these form a system of non-homogeneous conservation laws. We construct a variational approximation scheme that decreases the total mechanical energy and at the same time leads to physically realizable motions that avoid interpenetration of matter.
\end{abstract}

Key words. Elasticity, polyconvex, variational approximation.

AMS subject classifications. 35L70, 49J45, 74B20, 74H20.

\section{Introduction}

The equations describing radial motions of nonlinear, isotropic, elastic materials take the form

$$
w_{t t}=\frac{1}{R^{2}} \frac{\partial}{\partial R}\left(R^{2} \frac{\partial \Phi}{\partial v_{1}}\left(w_{R}, \frac{w}{R}, \frac{w}{R}\right)\right)-\frac{1}{R} \sum_{i=2}^{3} \frac{\partial \Phi}{\partial v_{i}}\left(w_{R}, \frac{w}{R}, \frac{w}{R}\right) .
$$

Here, $y$ stands for a radial motion $y(x, t)=w(R, t) \frac{x}{R}, R=|x|, x \in \mathbb{R}^{3}$, and (1.1) monitors the evolution of its amplitude $w(R, t)$. A necessary condition for $y$ to represent a physically realizable motion is $\operatorname{det} F>0$ with $F=\nabla y$. In the radial case, it dictates

$$
w_{R}(w / R)^{2}>0
$$

and is also a sufficient condition for avoiding interpenetration of matter.

The constitutive properties of hyperelastic materials are completely determined by the stored energy function $W(F): M_{+}^{3 \times 3} \rightarrow[0, \infty)$, which — due to frame indifference - has to be invariant under rotations. For isotropic elastic materials $W(F)=\Phi\left(v_{1}, v_{2}, v_{2}\right)$, where $\Phi$ is a symmetric function of the principal stretches $v_{1}, v_{2}, v_{3}$ of $F$; see [14]. Convexity of the stored energy is, in general, incompatible with certain physical requirements and is not a natural assumption. For instance, in order to avoid interpenetration of matter the stored energy should increase without bound as $\operatorname{det} F \rightarrow 0^{+}$so that compression of a finite volume down to a point would cost infinite energy. This behavior is inconsistent with simultaneously requiring convexity and invariance of the stored energy under rotations. As an alternative, the assumption of polyconvexity [1] is often employed, which postulates that

$$
W(F)=\sigma(F, \operatorname{cof} F, \operatorname{det} F)
$$

*Received: October 27, 2010; accepted (in revised version): March 9, 2011.

Research partially supported by the EU FP7-REGPOT project "Archimedes Center for Modeling, Analysis and Computation" and the EU EST-project "Differential Equations and Applications in Science and Engineering".

${ }^{\dagger}$ Department of Mathematics, University of Maryland, College Park, USA (amiroshn@gmail.com).

$\ddagger$ Department of Applied Mathematics, University of Crete, Heraklion, Greece (tzavaras@tem. uoc.gr). 
with $\sigma$ a convex function of the null-Lagrangian vector $(F, \operatorname{cof} F, \operatorname{det} F)$, and encompasses certain physically realistic models (e.g. $[5$, Sec $4.9,4.10])$. In this work, we employ a specific form of polyconvex stored energy,

$$
\begin{aligned}
W(F) & =\Phi\left(v_{1}, v_{2}, v_{3}\right) \\
& =\phi\left(v_{1}\right)+\phi\left(v_{2}\right)+\phi\left(v_{3}\right)+g\left(v_{2} v_{3}\right)+g\left(v_{1} v_{3}\right)+g\left(v_{1} v_{2}\right)+h\left(v_{1} v_{2} v_{3}\right),
\end{aligned}
$$

where $\phi, g$, and $h$ are convex functions and $h(\delta) \rightarrow+\infty$ as $\delta \rightarrow 0+$.

Equation (1.1) may be recast as a system of inhomogeneous balance laws,

$$
\begin{aligned}
v_{t} & =\frac{1}{R^{2}} \frac{\partial}{\partial R}\left(R^{2} \frac{\partial \Phi}{\partial v_{1}}\left(u, \frac{w}{R}, \frac{w}{R}\right)\right)-\frac{1}{R} \sum_{i=2}^{3} \frac{\partial \Phi}{\partial v_{i}}\left(u, \frac{w}{R}, \frac{w}{R}\right), \\
u_{t} & =v_{R}, \\
w_{t} & =v,
\end{aligned}
$$

where $u=w_{R}$, and $v=w_{t}$. The system admits the entropy-entropy flux pair

$$
R^{2} \partial_{t}\left(\frac{v^{2}}{2}+\Phi\left(u, \frac{w}{R}, \frac{w}{R}\right)\right)-\partial_{R}\left(R^{2} v \frac{\partial \Phi}{\partial v_{1}}\left(u, \frac{w}{R}, \frac{w}{R}\right)\right)=0,
$$

which expresses the conservation of mechanical energy along smooth solutions. For polyconvex stored energies, the "entropy"

$$
\eta=\frac{1}{2} v^{2}+\Phi\left(u, \frac{w}{R}, \frac{w}{R}\right)
$$

is not convex, which causes various difficulties in applying the general theory of conservation laws. Nevertheless, for three-dimensional elastodynamics there are available nonlinear transport identities for the null-Lagrangians [11] which allow us to view the equations of elasticity as constrained evolution of an enlarged symmetrizable system $[8,6]$ equipped with a relative entropy identity $[10]$. The enlarged system suggests a variational approximation scheme for polyconvex elasticity that dissipates the mechanical energy [8], and which, in the one-dimensional case, produces entropy weak solutions [7]. Conceptually similar structures are available in models of electromagnetism leading to augmented symmetrizable hyperbolic systems $[4,12,13]$.

The above results do not take into account the constraint of positive determinant, necessary to interpret $y$ as a physically realizable motion. In this article, we consider the equations of radial elasticity (1.1) and proceed to devise a variational approximation scheme that on one hand preserves the positivity of determinants (1.2) and on the other produces a time-discretized variant of entropy dissipation. As in [8], the scheme is based on transport identities for the null-Lagrangians. Null-Lagrangians are potential energies $\Psi\left(v_{1}, v_{2}, v_{3} ; R\right)$ for which the functional

$$
I[w]=\int_{0}^{1} \Psi\left(\left(w_{R}, \frac{w}{R}, \frac{w}{R}\right) ; R\right) d R
$$

has variational derivative zero. They satisfy

$$
-\partial_{R}\left(\Psi_{, 1}\right)+R^{-1}\left(\Psi_{, 2}+\Psi_{, 3}\right)=0 \text { for all functions } w(R),
$$


where $\Psi_{, i}:=\frac{\partial \Psi}{\partial v_{i}}, i=1,2,3$, stands for the partial derivative. The null-Lagrangians are computed to be the functions $v_{1}, v_{1} v_{2} R, v_{1} v_{3} R$, or $v_{1} v_{2} v_{3} R^{2}$. Along solutions of the dynamical problem, each null-Lagrangian satisfies the transport identity

$$
\partial_{t} \Psi=\partial_{R}(\Psi, 1 v)
$$

with $\Psi$ and $\Psi_{, i}$ are evaluated at $\Gamma=\left(w_{R}, w / R, w / R, R\right)$. The identities (1.8) allow us to embed the system (1.4) into the symmetrizable first-order evolution (3.6) in Section 3.2 .

The enlarged system, in the form (3.6), cannot handle the positivity of determinants constraint. For this reason we follow an alternative strategy, combining a change of variables suggested in Ball [3] (for the equilibrium problem) with the idea of extensions based on null-Lagrangians, and carry out an alternative extended system. We set $\rho=R^{3}, \alpha=w^{3}, \beta=w_{R} / R^{2}, \gamma=w^{2}$ and let

$$
\Xi=\left(\beta \rho^{2 / 3}, \frac{\alpha}{\rho}, \frac{\alpha}{\rho}, \frac{\gamma}{\rho^{1 / 3}}, \frac{3 \gamma_{\rho}}{2} \rho^{2 / 3}, \frac{3 \gamma_{\rho}}{2} \rho^{2 / 3}, \alpha_{\rho} \rho^{2 / 3}\right) .
$$

The second extension has four actual unknowns $v, \alpha, \beta$, and $\gamma$, and is the symmetrizable system listed in (3.26) of Section 3.3 endowed with the entropy pair

$$
\partial_{t}\left(\frac{v^{2}}{2}+G(\Xi)\right)-\partial_{\rho}\left(3 \rho^{2 / 3} G_{, i}(\Xi) \Omega_{, 1}^{i}(\Gamma) v\right)=0,
$$

where $G$ is defined in (3.12) and is (assumed) convex and $\Gamma$ is as in (3.14).

The extended system (3.26) is discretized in time using an implicit-explicit scheme. It is the Euler-Lagrange equations of the variational problem: given $v_{0}$ and $\Xi^{0}$ defined via $\alpha_{0}, \beta_{0}$, and $\gamma_{0}$ as in (1.9), minimize

$$
I(\alpha, \beta, \gamma, v)=\int_{0}^{1}\left\{\frac{1}{2}\left(v-v_{0}\right)^{2}+G(\Xi)\right\} d \rho
$$

over the set of admissible functions

$$
\begin{gathered}
\mathcal{A}_{\lambda}=\left\{(\alpha, \beta, \gamma, v) \in X: \alpha(0) \geqslant 0, \alpha(1)=\lambda, \alpha^{\prime}>0\right. \text { a.e. and } \\
I(\alpha, \beta, \gamma, v)<\infty, \frac{\left(\beta-\beta_{0}\right)}{h}=3 v^{\prime}, \\
\left.\frac{\left(\alpha-\alpha_{0}\right)}{h}=3 \alpha_{0}{ }^{2 / 3} v, \frac{\left(\gamma-\gamma_{0}\right)}{h}=2 \alpha_{0}{ }^{1 / 3} v\right\} .
\end{gathered}
$$

The differential constraints in (1.12) are affine, the condition $\alpha(1)=\lambda$ corresponds to the imposed boundary condition $y(x)=\lambda x, x \in \partial \mathcal{B}$, while $\alpha^{\prime}>0$ secures the positivity of determinants (1.2). We prove the existence and uniqueness of a minimizer for the functional $I$ over $\mathcal{A}_{\lambda}$ and that the minimizer is a weak solution to the corresponding Euler-Lagrange equations, that is, a solution of the time-discrete scheme. The analysis of the minimization problem (1.11)-(1.12) uses direct methods of the calculus of variations, in the spirit of [3], with the novel element of accounting for the evolutionary constraints in (1.12).

In continuum physics, weak solutions of a system of conservation laws are required to satisfy entropy inequalities of the form

$$
\partial_{t} \eta+\partial_{\alpha} q_{\alpha} \leq 0
$$


Such inequalities reflect irreversibility and originate from the second law of thermodynamics. For instance, admissible shocks of the elasticity equations are required to dissipate the mechanical energy. Accordingly, approximating schemes are expected to respect such behaviors and produce entropy dissipating solutions in the limit. The variational scheme studied here turns out to satisfy a discrete version of the entropy inequality

$$
\frac{\left(\frac{v^{2}}{2}+G(\Xi)\right)-\left(\frac{v_{0}^{2}}{2}+G\left(\Xi^{0}\right)\right)}{h}-\frac{d}{d \rho}\left(3 \rho^{2 / 3} G_{, i}(\Xi) \Omega_{, 1}^{i}\left(\Gamma^{0}\right) v\right) \leqslant 0
$$

(see Section 4). In addition, the approximants satisfy $\alpha_{\rho}>0$ - the transformed version of (1.2). Finally, if the constructed approximants converge pointwise as the time-step $h \rightarrow 0$, then the limit will satisfy the mechanical energy dissipation inequality

$$
\partial_{t}\left(\frac{v^{2}}{2}+G(\Xi)\right)-\partial_{\rho}\left(3 \rho^{2 / 3} G_{, i}(\Xi) \Omega_{, 1}^{i}(\Gamma) v\right) \leqslant 0 .
$$

The paper is organized as follows. In Section 2 we outline the derivation of the equations of radial elasticity and list various mechanical considerations relevant to this work. Section 3 contains a discussion of null-Lagrangians and the properties of the two symmetrizable extensions of (1.4) pursued. Section 4 introduces the timediscrete scheme and its relation to a variational problem. In Section 5 we consider the minimization problem (1.11) and prove Theorems 5.4 and 5.5 regarding existence and uniqueness of minimizers. The Euler-Lagrange equations associated to the minimization problem are derived in Theorem 6.1 of Section 6 , and the regularity of minimizers is discussed in Section 7. The fact that minimizers satisfy the time-discretized version of the entropy dissipation inequality (1.14) is proved in Section 4.

\section{Preliminaries}

We consider the equations of nonlinear elasticity

$$
\left\{\begin{aligned}
y_{t t} & =\operatorname{div} S(\nabla y), & & \text { in } \mathcal{B} \times(0, \infty), \\
y(x, t) & =\lambda x, & & \text { on } \partial \mathcal{B} \times[0, \infty), \\
\operatorname{det} \nabla y & >0, & & (x, t) \in \mathcal{B} \times[0, \infty),
\end{aligned}\right.
$$

on the unit ball $\mathcal{B}=\left\{x \in \mathbb{R}^{n}:|x|<1\right\}$, subject to uniform stretching at the boundary and initial conditions

$$
y(x, 0)=y_{0}(x), \quad y_{t}(x, 0)=v_{0}(x), \quad x \in \mathcal{B} .
$$

In order for the geometric mapping $y: \mathcal{B} \times[0, \infty) \rightarrow \mathbb{R}^{n}$ to correspond to a physically realizable motion we have to exclude interpenetration of matter. As a minimum requirement the condition $\operatorname{det} \nabla y>0$ is imposed.

Let $M^{n \times n}$ be the real $n \times n$ matrices, $M_{+}^{n \times n}=\left\{F \in M^{n \times n}: \operatorname{det} F>0\right\}$, and let $S O(n)$ denote the set of proper rotations. The Piola-Kirchhoff stress is a mapping $S: M_{+}^{n \times n} \rightarrow M^{n \times n}$, and for hyperelastic materials it is defined by the formula

$$
S(F)=\partial W(F) / \partial F
$$

where $W: M_{+}^{n \times n} \rightarrow \mathbb{R}^{n}$ is the stored-energy function of the elastic body. 
We assume that the stored energy function $W$ satisfies the physical requirement of frame-indifference and that the elastic material is isotropic. Then,

$$
W(Q F)=W(F)=W(F Q) \quad \forall F \in M_{+}^{n \times n}, Q \in S O(n)
$$

and (see Truesdell and Noll [14, pp 28, 317]) there exists a symmetric function

$$
\Phi: \mathbb{R}_{+}^{n}=\left\{x \in \mathbb{R}^{n}: x_{i}>0 \forall i\right\} \rightarrow \mathbb{R}
$$

such that

$$
W(F)=\Phi\left(v_{1}, \ldots, v_{n}\right) \quad \forall F \in M_{+}^{n \times n},
$$

where $v_{1}, \ldots, v_{n}$ are the singular values of $F$, i.e. the eigenvalues of $\left(F^{\mathrm{T}} F\right)^{1 / 2}$. We note that the symmetry of $\Phi$ implies

$$
\frac{\partial \Phi}{\partial v_{i}}(a, b, \ldots, b)=\frac{\partial \Phi}{\partial v_{j}}(a, b, \ldots, b), i, j \geqslant 2, a, b \in \mathbb{R}_{+} .
$$

It is easy to check that for hyperelastic, isotropic materials, frame-indifference implies

$$
S\left(Q F Q^{\mathrm{T}}\right)=Q S(F) Q^{\mathrm{T}}, \quad \text { for all } Q \in S O(n) .
$$

2.1. Radial elasticity. A function $f: \mathcal{B} \backslash\{0\} \rightarrow \mathbb{R}^{n}$ is called radial if

$$
f(x)=w(R) \frac{x}{R}, R=|x|,
$$

where $w:[0, \infty) \rightarrow[0, \infty)$. The space of deformations of $\mathcal{B}$ is denoted by

$$
\operatorname{Def}^{p}(\mathcal{B})=\left\{f \in W_{1}^{p}\left(\mathcal{B}, \mathbb{R}^{n}\right): \operatorname{det} \nabla f>0 \text { a.e. }\right\}
$$

Lemma 2.1 (J. Ball [3]). Let $f$ be a radial function. Then $f \in \operatorname{Def}^{p}(\mathcal{B})$ if and only if $w$ is absolutely continuous on $(0,1)$ and satisfies $w_{R}(w / R)^{n-1}>0$ almost everywhere, and

$$
\int_{0}^{1}\left(\left|w^{\prime}\right|^{p}+|w / R|^{p}\right) R^{n-1} d R<\infty .
$$

In this case the weak derivatives of $f$ are given by

$$
\nabla f=\frac{w}{R} \mathbf{I}+\left(w^{\prime}-\frac{w}{R}\right) \frac{x \otimes x}{R^{2}} \quad \text { a.e. } \quad x \in \mathcal{B} .
$$

Our next goal is to consider the problem (2.1) and to recast it for radial motions

$$
y(x, t)=w(R, t) \frac{x}{R} \quad \text { for } \quad x \neq 0,
$$

where $w:[0,1) \times[0, \infty) \rightarrow \mathbb{R}$ satisfies $w(R, t) \geqslant 0$. Lemma 2.1 implies

$$
\nabla y=\frac{w}{R} \mathbf{I}+\left(w_{R}-\frac{w}{R}\right) \frac{x \otimes x}{R^{2}} \quad \text { a.e. } \quad x \in \mathcal{B},
$$


and hence the eigenvalues of $\nabla y$ are expressed as

$$
v_{1}=w_{R}, v_{2}=\ldots=v_{n}=w / R \text {. }
$$

The requirement

$$
\operatorname{det} \nabla y=w_{R}(w / R)^{n-1}>0
$$

dictates $w_{R}, \frac{w}{R}>0$. Since $\nabla y$ is symmetric and positive definite, the singular values of $\nabla y$ coincide with its eigenvalues, the stored energy takes the form

$$
W(\nabla y)=\Phi\left(w_{R}, \frac{w}{R}, \ldots, \frac{w}{R}\right)
$$

and property (2.7) implies that the Piola-Kirchhoff stress can be expressed as (see e.g. J.Ball [3])

$$
\begin{aligned}
S(\nabla y)=\Phi_{, 2} & \left(w_{R}, w / R, \ldots, w / R\right) \mathbf{I} \\
& +\left[\Phi_{, 1}\left(w_{R}, w / R, \ldots, w / R\right)-\Phi_{, 2}\left(w_{R}, w / R, \ldots, w / R\right)\right] \frac{x \otimes x}{R^{2}}
\end{aligned}
$$

where $\Phi_{, j}:=\frac{\partial \Phi}{\partial v_{j}}, j=1,2,3$. For radial motions, the system $(2.1)$ then takes the form

$$
\begin{aligned}
R^{n-1} \partial_{t t} w & =\frac{\partial}{\partial R}\left(R^{n-1} \Phi_{, 1}\left(w_{R}, \ldots, w / R\right)\right)-R^{n-2} \sum_{i=2}^{n} \Phi_{, i}\left(w_{R}, \ldots, w / R\right) \\
w(1, t) & =\lambda, \quad w_{R}(w / R)^{n-1}>0,(R, t) \in(0,1) \times[0, \infty)
\end{aligned}
$$

of a second order equation describing the evolution of $w(R, t)$ subject to the constraint $(2.11)_{2}$. The latter expresses the requirement that matter cannot interpenetrate unto itself.

2.2. Polyconvex stored energy for $n=3$. From now on we fix the number of dimensions to $n=3$ and assume that the stored energy $W: M_{+}^{3 \times 3} \rightarrow \mathbb{R}^{3}$ is polyconvex, that is,

$$
W(F)=\overline{\bar{G}}(F, \operatorname{cof} F, \operatorname{det} F)
$$

for some convex function $\overline{\bar{G}}: M_{+}^{3 \times 3} \times M_{+}^{3 \times 3} \times \mathbb{R}_{+} \rightarrow \mathbb{R}$.

By the polar decomposition theorem any matrix $F \in M_{+}^{3 \times 3}$ is expressed in the form $F=R U$ with $R \in S O(3)$ and $U=+\sqrt{F^{T} F}$. Further, $U=Q^{T} \operatorname{diag}\left(v_{1}, v_{2}, v_{3}\right) Q$ where $Q$ is the orthogonal matrix of eigenvectors and $v_{1}, v_{2}, v_{3}$ are the eigenvalues of $U$. The properties (2.4) of isotropy and frame-indifference imply

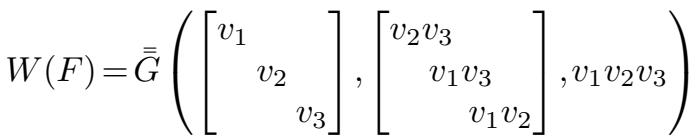

$$
\begin{aligned}
& =: \bar{G}\left(v_{1}, v_{2}, v_{3}, v_{2} v_{3}, v_{1} v_{3}, v_{1} v_{2}, v_{1} v_{2} v_{3}\right)
\end{aligned}
$$

where $\bar{G}(\Xi)$ is a convex function of $\Xi=(\xi)_{i=1 \ldots 7} \in \mathbb{R}^{7}$. 
For radial motions the singular values are $v_{1}=w_{R}, v_{2}=v_{3}=\frac{w}{R}$. For reasons related to the null-Lagrangian structure of an associated variational problem (outlined in the following section) the stored energy will be expressed in the form

$$
\begin{aligned}
W(\nabla y) & =\Phi\left(w_{R}, \frac{w}{R}, \frac{w}{R}\right) \\
& =\bar{G}\left(w_{R}, \frac{w}{R}, \frac{w}{R},\left(\frac{w}{R}\right)^{2}, w_{R}\left(\frac{w}{R}\right), w_{R}\left(\frac{w}{R}\right), w_{R}\left(\frac{w}{R}\right)^{2}\right) \\
& =G\left(\Omega\left(\left(w_{R}, \frac{w}{R}, \frac{w}{R}\right) ; R\right) ; R\right),
\end{aligned}
$$

where $\Omega$ and $G$ are inhomogeneous functions defined by

$$
\begin{aligned}
& \Omega(V ; R):=\left(v_{1}, v_{2}, v_{3}, v_{2} v_{3} R, v_{1} v_{3} R, v_{1} v_{2} R, v_{1} v_{2} v_{3} R^{2}\right) \\
& G(\Xi ; R):=\bar{G}\left(\xi_{1}, \xi_{2}, \xi_{3}, \xi_{4} / R, \xi_{5} / R, \xi_{6} / R, \xi_{7} / R^{2}\right)
\end{aligned}
$$

$V=\left(v_{i}\right)_{i=1 \ldots 3} \in \mathbb{R}^{3}$, and $\Xi=(\xi)_{i=1 \ldots 7} \in \mathbb{R}^{7}$. The convexity hypothesis on $\overline{\bar{G}}$ implies that $G(\Xi ; R)$ is convex as a function of $\Xi \in \mathbb{R}^{7}$. In summary,

$$
\begin{aligned}
W(\nabla y)=\Phi\left(w_{R}, \frac{w}{R}, \frac{w}{R}\right) & =G(\Omega(\Gamma ; R) ; R), \\
\text { where } \quad \Gamma & =\left(w_{R}, \frac{w}{R}, \frac{w}{R}\right) .
\end{aligned}
$$

For simplicity of notation, we henceforth suppress the dependence on $R$ and write $\Omega(V)=\Omega(V ; R)$ and $G(\Xi)=G(\Xi ; R)$.

Equation (2.11) can be expressed in the form

$$
\begin{aligned}
R^{2} \partial_{t} v & =\frac{\partial}{\partial R}\left(R^{2} \Phi_{, 1}\left(w_{R}, \frac{w}{R}, \frac{w}{R}\right)\right)-R\left(\Phi_{, 2}+\Phi_{, 3}\right)\left(w_{R}, \frac{w}{R}, \frac{w}{R}\right), \\
\partial_{t} w & =v
\end{aligned}
$$

The latter formally satisfies the conservation of mechanical energy identity

$$
\partial_{t}\left(R^{2}\left(\frac{v^{2}}{2}+\Phi\left(w_{R}, w / R, w / R\right)\right)\right)=\partial_{R}\left(R^{2} v \Phi_{, 1}\left(w_{R}, w / R, w / R\right)\right)
$$

The mechanical energy and the associated energy flux provide an entropy-entropy flux pair for (2.17), but the entropy is not in general convex. Using (2.15)-(2.16), the derivatives $\Phi_{, j}$ are expressed as

$$
\Phi_{, j}\left(v_{1}, v_{2}, v_{3}\right)=\frac{\partial}{\partial v_{j}} G(\Omega(V))=\frac{\partial G}{\partial \xi_{i}}(\Omega(V)) \frac{\partial \Omega^{i}}{\partial v_{j}}(V),
$$

and $(2.17)_{1}$ is written as

$$
\begin{aligned}
R^{2} \partial_{t} v= & \partial_{R}\left(R^{2} \frac{\partial G}{\partial \xi_{i}}(\Omega(\Gamma)) \frac{\partial \Omega^{i}}{\partial v_{1}}(\Gamma)\right) \\
& -R \frac{\partial G}{\partial \xi_{i}}(\Omega(\Gamma))\left(\frac{\partial \Omega^{i}}{\partial v_{2}}(\Gamma)+\frac{\partial \Omega^{i}}{\partial v_{3}}(\Gamma)\right) .
\end{aligned}
$$




\section{Null-Lagrangians and extensions of polyconvex radial elasticity}

3.1. Null-Lagrangians. An alternative approach to derive (2.18) proceeds by considering the extrema of the action functional

$$
J[y]=\int_{0}^{T} \int_{0}^{1}\left(\frac{1}{2} w_{t}^{2}-\Phi\left(w_{R}, \frac{w}{R}, \frac{w}{R}\right)\right) R^{2} d R d t
$$

and deriving (2.11) (for $n=3$ ) as the associated Euler-Lagrange equations. This provides a connection with the calculus of variations.

Consider the functional associated to the equilibrium problem

$$
I[w]=\int_{0}^{1} \Psi\left(w_{R}, w / R, w / R ; R\right) d R .
$$

We ask for which integrands $\Psi\left(v_{1}, v_{2}, v_{3} ; R\right): \mathbb{R}^{4} \rightarrow \mathbb{R}$ the functional $I$ admits zero variational derivatives, $\frac{\delta I}{\delta w}=0$; such integrands are called null Lagrangians and they satisfy the Euler-Lagrange equation

$$
-\partial_{R}\left(\Psi_{, 1}\right)+R^{-1}\left(\Psi_{, 2}+\Psi_{, 3}\right)=0 \text { for all functions } w(R) .
$$

If $w=w(R, t)$ also depends on time, the evolution of a null Lagrangian $\Psi$ is described by

$$
\partial_{t} \Psi=\partial_{R}\left(\Psi_{, 1} \partial_{t} w\right)
$$

where $\Psi$ and $\Psi_{, i}$ are evaluated at $\left(w_{R}, w / R, w / R, R\right)$.

It is easily verified that $\Psi\left(v_{1}, v_{2}, v_{3} ; R\right)$, selected by

$$
v_{1}, v_{1} v_{2} R, v_{1} v_{3} R, \quad \text { or } \quad v_{1} v_{2} v_{3} R^{2}
$$

are null-Lagrangians. Applying (3.1) to $\Omega^{i}, i=1,5,6,7$, defined by (2.13), we get

$$
-\partial_{R}\left(\Omega_{, 1}^{i}(\Gamma)\right)+R^{-1}\left(\Omega_{, 2}^{i}(\Gamma)+\Omega_{, 3}^{i}(\Gamma)\right)=0, \quad i=1,5,6,7,
$$

with $\Gamma=\left(w_{R}, w / R, w / R\right)$ defined by $(2.16)$.

3.2. A symmetrizable extension. The null-Lagrangian structure is used in [8] to embed the equations of 3-d elastodynamics to a hyperbolic system endowed with a convex entropy, and to construct a variational approximation scheme for the problem. We follow this procedure in order to achieve an augmented system for radial elastodynamics. The evolution in time of

$$
\Omega(\Gamma)=\left(w_{R}, w / R, w / R, w^{2} / R, w_{R} w, w_{R} w, w_{R} w^{2}\right)
$$

gives

$$
\begin{aligned}
& \partial_{t} \Omega^{1}(\Gamma)=\partial_{t}\left(w_{R}\right)=\partial_{R} v=\partial_{R}\left(\Omega_{, 1}^{1}(\Gamma) v\right), \\
& \partial_{t} \Omega^{i}(\Gamma)=\partial_{t}(w / R)=v / R=R^{-1}\left(\Omega_{, 2}^{i}(\Gamma)+\Omega_{, 3}^{i}(\Gamma)\right) v \quad \text { for } i=2,3, \\
& \partial_{t} \Omega^{4}(\Gamma)=\partial_{t}\left(w^{2} / R\right)=2(w / R) v=R^{-1}\left(\Omega_{, 2}^{4}(\Gamma)+\Omega_{, 3}^{4}(\Gamma)\right) v, \\
& \partial_{t} \Omega^{i}(\Gamma)=\partial_{t}\left(w_{R} w\right)=\partial_{R}(w v)=\partial_{R}\left(\Omega_{, 1}^{i}(\Gamma) v\right) \quad \text { for } i=5,6, \\
& \partial_{t} \Omega^{7}(\Gamma)=\partial_{t}\left(w_{R} w^{2}\right)=\partial_{R}\left(w^{2} v\right)=\partial_{R}\left(\Omega_{, 1}^{7}(\Gamma) v\right) .
\end{aligned}
$$


Note that (3.5) $1,5,6,7$ are precisely the equations (3.2) describing the evolution of null Lagrangians. By contrast, (3.5) $)_{2,3,4}$ describe the evolution of lower-order terms and do not have the structure of $(3.2)$.

Equations (3.5) and (2.19) motivate an extension of radial elasticity:

$$
\left\{\begin{aligned}
R^{2} \partial_{t} v & =\partial_{R}\left(R^{2} \frac{\partial G}{\partial \xi_{i}}(\Xi) \frac{\partial \Omega^{i}}{\partial v_{1}}(\Gamma)\right)-R \frac{\partial G}{\partial \xi_{i}}(\Xi)\left(\frac{\partial \Omega^{i}}{\partial v_{2}}(\Gamma)+\frac{\partial \Omega^{i}}{\partial v_{3}}(\Gamma)\right), \\
\partial_{t} w & =v \\
\partial_{t} \xi_{i} & =\partial_{R}\left(\Omega_{, 1}^{i}(\Gamma) v\right) i=1,5,6,7 \\
\partial_{t} \xi_{i} & =R^{-1}\left(\Omega_{, 2}^{i}(\Gamma)+\Omega_{, 3}^{i}(\Gamma)\right) v i=2,3,4
\end{aligned}\right.
$$

where $\Gamma=\left(w_{R}, \frac{w}{R}, \frac{w}{R}\right)$, subject to the constraints

$$
\xi_{2}, \xi_{3}>0, \xi_{7}>0,(R, t) \in(0,1) \times(0, \infty),
$$

and the boundary conditions $w(1, t)=\xi_{2}(1, t)=\xi_{3}(1, t)=\lambda$. System (3.6) describes the evolution of the vector $(v, w, \Xi)$, with $\Xi \in \mathbb{R}^{7}$, and is provided with initial data $\left(v_{0}, w_{0}, \Xi_{0}\right)$.

The extension has the following properties:

(i) If $\Xi(\cdot, 0)=\Omega\left(\Gamma^{0}\right)$ where $\Gamma^{0}=\left(w_{0}^{\prime}, w_{0} / R, w_{0} / R\right)$, then $\Xi(R, t)=\Omega(\Gamma(R, t))$, where $\Gamma=$ $\left(w_{R}, w / R, w / R\right)$. In other words, radial elasticity $(2.17)$ can be viewed as a constrained evolution of (3.6).

(ii) The enlarged system admits an entropy pair

$$
\partial_{t}\left(R^{2}\left(\frac{v^{2}}{2}+G(\Xi)\right)\right)-\partial_{R}\left(R^{2} \frac{\partial G}{\partial \xi_{i}}(\Xi) \frac{\partial \Omega^{i}}{\partial v_{1}}(\Gamma) v\right)=0,
$$

with strictly convex entropy

$$
\eta(v, \Xi)=\frac{v^{2}}{2}+G(\Xi) .
$$

The identity (3.8) holds for general solutions $(v, w, \Xi)$ of (3.6) and is derived upon using the property (3.3) for the null Lagrangians (2.13).

3.3. An alternative extension with a convex entropy. System (3.6) provides an extension of radial elasticity that is endowed with a convex entropy. Concerning the objective of achieving a variational approximation, it has the drawback that the constraint (3.7) of positivity for the variables $\xi_{2}, \xi_{3}$, and $\xi_{7}$ is not preserved at the level of time-step approximations. Although one can control the positivity of $\xi_{7}$ (the augmented variable standing for the determinant), it is not possible to control the positivity of $\xi_{2}, \xi_{3}$. There are also difficulties in proving that minimizers satisfy the corresponding Euler-Lagrange equations, the time-discretized system associated to $(3.6)$.

For this reason, we develop an alternative extension by combining the evolution of null-Lagrangians with a change of variables used in Ball [3] for the equilibrium problem. This extension induces a variational approximation scheme that preserves the positivity of determinants.

The stored energy $\Phi$ is expressed in the form

$$
\begin{aligned}
\Phi\left(v_{1}, v_{2}, v_{3}\right) & =\bar{G}\left(v_{1}, v_{2}, v_{3}, v_{2} v_{3}, v_{1} v_{3}, v_{1} v_{2}, v_{1} v_{2} v_{3}\right) \\
& =G(\Omega(V ; \rho) ; \rho),
\end{aligned}
$$


where $\Omega$ and $G$ are nonhomogeneous functions of $\rho$ that are redefined so that

$$
\begin{aligned}
& \Omega(V ; \rho):=\left(v_{1}, v_{2}^{3}, v_{3}^{3}, v_{2} v_{3} \rho^{1 / 3}, v_{1} v_{3} \rho^{1 / 3}, v_{1} v_{2} \rho^{1 / 3}, v_{1} v_{2} v_{3} \rho^{2 / 3}\right), \\
& G(\Xi ; \rho):=\bar{G}\left(\xi_{1}, \xi_{2}^{1 / 3}, \xi_{3}^{1 / 3}, \xi_{4} / \rho^{1 / 3}, \xi_{5} / \rho^{1 / 3}, \xi_{6} / \rho^{1 / 3}, \xi_{7} / \rho^{2 / 3}\right) .
\end{aligned}
$$

It is now assumed that $G(\Xi ; \rho)$ is a convex function of $\Xi$; this is a somewhat stronger hypothesis than polyconvexity (which is convexity of $\bar{G}$ ) because of the definition of $\Omega^{i}(V ; \rho), i=2,3$, in (3.11). In the sequel any explicit $\rho$-dependence will be suppressed.

3.3.1. A change of variables. Following [3] we perform the change of variables

$$
\rho=R^{3} \quad \text { and } \quad \alpha=w^{3} .
$$

Then $\Gamma=\left(w_{R}, w / R, w / R\right)$ is expressed as

$$
\Gamma=\left(\alpha_{\rho}(\rho / \alpha)^{2 / 3},(\alpha / \rho)^{1 / 3},(\alpha / \rho)^{1 / 3}\right)
$$

and the stored energy reads

$$
\begin{aligned}
W(\nabla y) & =\Phi\left(\alpha_{\rho}(\rho / \alpha)^{2 / 3},(\alpha / \rho)^{1 / 3},(\alpha / \rho)^{1 / 3}\right) \\
& =G(\Omega(\Gamma ; \rho) ; \rho),
\end{aligned}
$$

where $\Omega$ and $G$ are defined in (3.11), (3.12), and $G(\cdot ; \rho)$ is convex.

The system (2.17) takes the form

$$
\left\{\begin{aligned}
\partial_{t} v= & \partial_{\rho}\left(3 \rho^{2 / 3} \frac{\partial G}{\partial \xi_{i}}(\Omega(\Gamma)) \frac{\partial \Omega^{i}}{\partial v_{1}}(\Gamma)\right) \\
& -\rho^{-1 / 3} \frac{\partial G}{\partial \xi_{i}}(\Omega(\Gamma))\left(\frac{\partial \Omega^{i}}{\partial v_{2}}(\Gamma)+\frac{\partial \Omega^{i}}{\partial v_{3}}(\Gamma)\right), \\
\partial_{t}\left(\alpha^{1 / 3}\right) & =v \\
\alpha(1) & =\lambda, \alpha \geqslant 0, \alpha_{\rho}>0,(R, t) \in(0,1) \times[0, \infty),
\end{aligned}\right.
$$

with the last inequalities encoding the constraints for solutions to represent elastic motions. In the new variables, by (3.11),

$$
\Omega(\Gamma)=\left(\frac{\alpha_{\rho}}{\alpha^{2 / 3}} \rho^{2 / 3}, \frac{\alpha}{\rho}, \frac{\alpha}{\rho}, \frac{\alpha^{2 / 3}}{\rho^{1 / 3}}, \frac{\alpha_{\rho}}{\alpha^{1 / 3}} \rho^{2 / 3}, \frac{\alpha_{\rho}}{\alpha^{1 / 3}} \rho^{2 / 3}, \alpha_{\rho} \rho^{2 / 3}\right)
$$

and, using $(3.16)_{2}$, we compute

$$
\begin{aligned}
& \partial_{t} \Omega^{1}(\Gamma)=\partial_{t}\left(3 \rho^{2 / 3} \partial_{\rho}\left(\alpha^{1 / 3}\right)\right)=3 \rho^{2 / 3} \partial_{\rho} v, \\
& \partial_{t} \Omega^{i}(\Gamma)=\partial_{t}(\alpha / \rho)=3 \alpha^{2 / 3} v / \rho \quad i=2,3, \\
& \partial_{t} \Omega^{4}(\Gamma)=\partial_{t}\left(\alpha^{2 / 3} / \rho^{1 / 3}\right)=2 \alpha^{1 / 3} v / \rho^{1 / 3}, \\
& \partial_{t} \Omega^{i}(\Gamma)=\partial_{t}\left((3 / 2) \rho^{2 / 3} \partial_{\rho}\left(\alpha^{2 / 3}\right)\right)=3 \rho^{2 / 3} \partial_{\rho}\left(\alpha^{1 / 3} v\right) \quad i=5,6, \\
& \partial_{t} \Omega^{7}(\Gamma)=\partial_{t}\left(\alpha_{\rho} \rho^{2 / 3}\right)=3 \rho^{2 / 3} \partial_{\rho}\left(\alpha^{2 / 3} v\right) .
\end{aligned}
$$


These identities are summarized in two groups as

$$
\begin{aligned}
& \partial_{t} \Omega^{i}(\Gamma)=3 \rho^{2 / 3} \partial_{\rho}\left(\Omega_{, 1}^{i}(\Gamma) v\right), i=1,5,6,7, \\
& \partial_{t} \Omega^{i}(\Gamma)=\rho^{-1 / 3}\left(\Omega_{, 2}^{i}(\Gamma)+\Omega_{, 3}^{i}(\Gamma)\right) v, i=2,3,4,
\end{aligned}
$$

the former representing the evolution of null-Lagrangians and the latter the evolution of lower order terms. The identities (3.3) satisfied by null-Lagrangians become

$$
-3 \rho^{2 / 3} \partial_{\rho}\left(\Omega_{, 1}^{i}(\Gamma)\right)+\rho^{-1 / 3}\left(\Omega_{, 2}^{i}(\Gamma)+\Omega_{, 3}^{i}(\Gamma)\right)=0, \quad i=1,5,6,7 .
$$

3.3.2. The augmented system. Next, consider the augmented system

$$
\left\{\begin{aligned}
\partial_{t} v & =\partial_{\rho}\left(3 \rho^{2 / 3} G_{, i}(\Xi) \Omega_{, 1}^{i}(\Gamma)\right)-\rho^{-1 / 3} G_{, i}(\Xi)\left(\Omega_{, 2}^{i}(\Gamma)+\Omega_{, 3}^{i}(\Gamma)\right), \\
\partial_{t} \alpha^{1 / 3} & =v \\
\partial_{t} \xi_{i} & =3 \rho^{2 / 3} \partial_{\rho}\left(\Omega_{, 1}^{i}(\Gamma) v\right), i=1,5,6,7 \\
\partial_{t} \xi_{i} & =\rho^{-1 / 3}\left(\Omega_{, 2}^{i}(\Gamma)+\Omega_{, 3}^{i}(\Gamma)\right) v, i=2,3,4
\end{aligned}\right.
$$

where $\Gamma$ is given by (3.14), subject to the boundary conditions and constraints, respectively,

$$
\alpha(1)=\lambda, \alpha \geqslant 0, \alpha_{\rho}>0,(\rho, t) \in(0,1) \times(0, \infty) .
$$

The system $(3.21)_{1}-(3.21)_{4}$ is a second-order system describing the evolution of the vector $(v, \alpha, \Xi)$ and is assigned initial data $\left(v_{0}, \alpha_{0}, \Xi_{0}\right)$. It has the following properties:

(a) If $\Xi(\cdot, 0)=\Omega\left(\Gamma^{0}\right)$ with $\Gamma^{0}=\left(\alpha_{0}^{\prime}\left(\rho / \alpha_{0}\right)^{2 / 3},\left(\alpha_{0} / \rho\right)^{1 / 3},\left(\alpha_{0} / \rho\right)^{1 / 3}\right)$, then $\Xi=\Omega(\Gamma)$ for all times. In other words, radial elasticity $(2.11)$ can be viewed as a constrained evolution of (3.21).

(b) The enlarged system admits an entropy pair

$$
\partial_{t}\left(\frac{v^{2}}{2}+G(\Xi)\right)-\partial_{\rho}\left(3 \rho^{2 / 3} G_{, i}(\Xi) \Omega_{, 1}^{i}(\Gamma) v\right)=0
$$

with (for convex $G$ ) convex entropy $\eta(v, \Xi)=\frac{v^{2}}{2}+G(\Xi)$.

At this point we set

$$
\begin{gathered}
\beta=\alpha_{\rho} / \alpha^{2 / 3}, \quad \gamma=\alpha^{2 / 3} \\
\Xi=\left(\beta \rho^{2 / 3}, \frac{\alpha}{\rho}, \frac{\alpha}{\rho}, \frac{\gamma}{\rho^{1 / 3}}, \frac{3 \gamma_{\rho}}{2} \rho^{2 / 3}, \frac{3 \gamma_{\rho}}{2} \rho^{2 / 3}, \alpha_{\rho} \rho^{2 / 3}\right),
\end{gathered}
$$

and proceed to simplify the extended system working with $\alpha, \beta, \gamma, v$ as the independent variables.

Taking a closer look at the extended system we see that $\xi_{2}=\xi_{3}$ by construction and hence equations $(3.21)_{2}, i=2,3$ are identical. Moreover,

$$
\begin{aligned}
\partial_{t} \xi_{2}=3 \alpha^{2 / 3} v / \rho & \Rightarrow \partial_{t} \xi_{7}=\rho^{2 / 3} \partial_{\rho}\left(\rho \partial_{t} \xi_{2}\right) \\
\partial_{t} \xi_{4}=2 \alpha^{1 / 3} v / \rho^{1 / 3} & \Rightarrow \partial_{t} \xi_{5}=\partial_{t} \xi_{6}=\frac{3}{2} \rho^{2 / 3} \partial_{\rho}\left(\rho^{1 / 3} \partial_{t} \xi_{4}\right) .
\end{aligned}
$$


Hence (3.21) is overdetermined and extra equations $(3.21)_{2}, i=5,6,7$ and $(3.21)_{3}, i=3$ can be excluded. In explicit form the extension is written as

$$
\left\{\begin{aligned}
\partial_{t} v & =\partial_{\rho}\left(3 \rho^{2 / 3} G_{, i}(\Xi) \Omega_{, 1}^{i}(\Gamma)\right)-\rho^{-1 / 3} G_{, i}(\Xi)\left(\Omega_{, 2}^{i}(\Gamma)+\Omega_{, 3}^{i}(\Gamma)\right), \\
\partial_{t} \beta & =\partial_{\rho}(3 v) \\
\partial_{t} \alpha & =3 \alpha^{2 / 3} v \\
\partial_{t} \gamma & =2 \alpha^{1 / 3} v \\
\alpha(1) & =\lambda, \alpha \geqslant 0, \alpha_{\rho}>0,(\rho, t) \in(0,1) \times[0, \infty),
\end{aligned}\right.
$$

where from $(3.26)_{3}$ and $(3.26)_{4}$ we can derive the excluded equations

$$
\begin{aligned}
& \partial_{t} \alpha_{\rho}=\partial_{\rho}\left(3 \alpha^{2 / 3} v\right), \\
& \partial_{t} \gamma_{\rho}=\partial_{\rho}\left(2 \alpha^{1 / 3} v\right) .
\end{aligned}
$$

\section{Variational approximation scheme}

In this section we introduce a variational approximation scheme for the radial equation of elastodynamics. The general approach is to discretize the extended system by use of implicit-explicit scheme.

Successive iterates are constructed by discretizing (3.21) as follows: Given the $(j-1)^{t h}$ iterate $\left(\alpha_{0}, \beta_{0}, \gamma_{0}, v_{0}\right)$ with $\alpha_{0}(\rho) \geqslant 0$ and $\alpha_{0}^{\prime}(\rho)>0, \rho \in(0,1)$, we define $\Xi^{0}=$ $\left(\xi_{i}^{0}\right)_{i=1}^{7}$ by

$$
\Xi^{0}(\rho)=\left(\beta_{0} \rho^{2 / 3}, \frac{\alpha_{0}}{\rho}, \frac{\alpha_{0}}{\rho}, \frac{\gamma_{0}}{\rho^{1 / 3}}, \frac{3 \gamma_{0}^{\prime}}{2} \rho^{2 / 3}, \frac{3 \gamma_{0}^{\prime}}{2} \rho^{2 / 3}, \alpha_{0}^{\prime} \rho^{2 / 3}\right)
$$

and construct the $j^{\text {th }}$ iterate $(\alpha, \beta, \gamma, v)$, with corresponding $\Xi=\left(\xi_{i}\right)_{i=1}^{7}$ defined by

$$
\Xi(\rho)=\left(\beta \rho^{2 / 3}, \frac{\alpha}{\rho}, \frac{\alpha}{\rho}, \frac{\gamma}{\rho^{1 / 3}}, \frac{3 \gamma^{\prime}}{2} \rho^{2 / 3}, \frac{3 \gamma^{\prime}}{2} \rho^{2 / 3}, \alpha^{\prime} \rho^{2 / 3}\right),
$$

by solving

$$
\left\{\begin{aligned}
\left(v-v_{0}\right) / h= & \frac{d}{d \rho}\left(3 \rho^{2 / 3} G_{, i}(\Xi) \Omega_{, 1}^{i}\left(\Gamma^{0}\right)\right) \\
& -\rho^{-1 / 3} G_{, i}(\Xi)\left(\Omega_{, 2}^{i}\left(\Gamma^{0}\right)+\Omega_{, 3}^{i}\left(\Gamma^{0}\right)\right), \\
\left(\xi_{i}-\xi_{i}^{0}\right) / h & =3 \rho^{2 / 3} \frac{d}{d \rho}\left(\Omega_{, 1}^{i}\left(\Gamma^{0}\right) v\right), i=1,5,6,7, \\
\left(\xi_{i}-\xi_{i}^{0}\right) / h & =\rho^{-1 / 3}\left(\Omega_{, 2}^{i}\left(\Gamma^{0}\right)+\Omega_{, 3}^{i}\left(\Gamma^{0}\right)\right) v, i=2,3,4, \\
\xi_{2}(1) & =\xi_{3}(1)=\lambda, \xi_{2}, \xi_{3} \geqslant 0, \xi_{7}>0, \rho \in(0,1),
\end{aligned}\right.
$$

where

$$
\begin{aligned}
\Gamma & =\left(\alpha^{\prime}(\rho / \alpha)^{2 / 3},(\alpha / \rho)^{1 / 3},(\alpha / \rho)^{1 / 3}\right), \\
\Gamma^{0} & =\left(\alpha_{0}^{\prime}\left(\rho / \alpha_{0}\right)^{2 / 3},\left(\alpha_{0} / \rho\right)^{1 / 3},\left(\alpha_{0} / \rho\right)^{1 / 3}\right) .
\end{aligned}
$$

As in the continuous case the discrete system (4.3) is overdetermined with extra equations

$$
\begin{aligned}
& \left(\alpha_{\rho}-\alpha_{0 \rho}\right) / h=\frac{d}{d \rho}\left(3 \alpha_{0}^{2 / 3} v\right), \\
& \left(\gamma_{\rho}-\gamma_{0}\right) / h=\frac{d}{d \rho}\left(2 \alpha_{0}^{1 / 3} v\right),
\end{aligned}
$$


corresponding to $(4.3)_{2}, i=5,6,7$. Excluding them from the system above we get

$$
\left\{\begin{aligned}
\left(v-v_{0}\right) / h= & \frac{d}{d \rho}\left(3 \rho^{2 / 3} G_{, i}(\Xi) \Omega_{, 1}^{i}\left(\Gamma^{0}\right)\right) \\
& -\rho^{-1 / 3} G_{, i}(\Xi)\left(\Omega_{, 2}^{i}\left(\Gamma^{0}\right)+\Omega_{, 3}^{i}\left(\Gamma^{0}\right)\right) \\
\left(\beta-\beta_{0}\right) / h= & \frac{d}{d \rho}(3 v) \\
\left(\alpha-\alpha_{0}\right) / h= & 3 \alpha_{0}{ }^{2 / 3} v \\
\left(\gamma-\gamma_{0}\right) / h= & 2 \alpha_{0}{ }^{1 / 3} v \\
\alpha(1)= & \lambda, \alpha \geqslant 0, \alpha^{\prime}>0, \rho \in(0,1)
\end{aligned}\right.
$$

Note that Equation (4.6) can be derived from $(4.7)_{3,4}$.

Time-step approximations capture a subtle form of dissipation associated with the underlying variational structure and the convexity of the entropy $[7,8]$. Indeed, solutions of (4.7) satisfy a discrete entropy inequality; to see that, consider a smooth solution $(\Xi, v)$ of $(4.3)$ associated to smooth data $\left(\Xi^{0}, v_{0}\right)$ given by (4.1). Multiplying $(4.3)_{1}$ by $v$ we get

$$
\begin{aligned}
& \frac{v\left(v-v_{0}\right)}{h}+G_{, i}(\Xi)\left(3 \rho^{2 / 3} \Omega_{, 1}^{i}\left(\Gamma^{0}\right) \frac{d v}{d \rho}+\rho^{-1 / 3}\left(\Omega_{, 2}^{i}\left(\Gamma^{0}\right)+\Omega_{, 3}^{i}\left(\Gamma^{0}\right)\right) v\right) \\
= & \frac{d}{d \rho}\left(3 \rho^{2 / 3} G_{, i}(\Xi) \Omega_{, 1}^{i}\left(\Gamma^{0}\right) v\right) .
\end{aligned}
$$

Then denoting

$$
A_{i}=3 \rho^{2 / 3} \Omega_{, 1}^{i}\left(\Gamma^{0}\right) \frac{d v}{d \rho}+\rho^{-1 / 3}\left(\Omega_{, 2}^{i}\left(\Gamma^{0}\right)+\Omega_{, 3}^{i}\left(\Gamma^{0}\right)\right) v, i=1, \ldots, 7
$$

we claim

$$
A_{i}=\frac{\xi_{i}-\xi_{i}^{0}}{h}
$$

Indeed, for $i=2,3,4$ we have $\Omega_{1}^{i}=0$ and hence (4.3) $)_{3}$ and (4.9) imply (4.10). For $i=1,5,6,7$, by the properties (3.20) of null Lagrangians and $(4.3)_{2}$ we get

$$
\begin{aligned}
A_{i}= & v\left(-3 \rho^{2 / 3} \frac{d}{d \rho}\left(\Omega_{, 1}^{i}\left(\Gamma^{0}\right)\right)+\rho^{-1 / 3}\left(\Omega_{, 2}^{i}\left(\Gamma^{0}\right)+\Omega_{, 3}^{i}\left(\Gamma^{0}\right)\right)\right) \\
& +3 \rho^{2 / 3} \frac{d}{d \rho}\left(\Omega_{, 1}^{i}\left(\Gamma^{0}\right) v\right)=\left(\xi_{i}-\xi_{i}^{0}\right) / h, i=1,5,6,7
\end{aligned}
$$

Thus (4.8) and (4.10) imply

$$
\frac{1}{h}\left(v\left(v-v_{0}\right)+G_{, i}(\Xi)\left(\xi_{i}-\xi_{i}^{0}\right)\right)=\frac{d}{d \rho}\left(3 \rho^{2 / 3} G_{, i}(\Xi) \Omega_{, 1}^{i}\left(\Gamma^{0}\right) v\right) .
$$

Now, we denote $\Theta=(v, \Xi)$ and $\Theta^{0}=\left(v_{0}, \Xi^{0}\right)$. Then $\eta=1 / 2 v^{2}+G(\Xi)$ satisfies

$$
\frac{1}{h} D \eta \cdot\left(\Theta-\Theta^{0}\right)-\frac{d}{d \rho}\left(3 \rho^{2 / 3} G_{, i}(\Xi) \Omega_{, 1}^{i}\left(\Gamma^{0}\right) v\right)=0 .
$$


For $G$ convex the following identity holds:

$$
\frac{\eta(\Theta)-\eta\left(\Theta^{0}\right)}{h}-\frac{d}{d \rho}\left(3 \rho^{2 / 3} G_{, i}(\Xi) \Omega_{, 1}^{i}\left(\Gamma^{0}\right) v\right) \leqslant 0 .
$$

REMARK 4.1. We have not studied in this article the convergence as the time-step $h \rightarrow 0$. For the three-dimensional elasticity equations this process produces measurevalued solutions [8] while for one-dimensional elasticity it gives entropy weak solutions [7]. In the present case we would expect to obtain weak solutions, but the compactness properties of (1.4) are not at present sufficiently understood. There are two differences of (1.4) relative to the well understood compactness theory of one-dimensional elasticity: the dependence of the stress on lower order terms, and the singularity at $R=0$. Nevertheless, if the iterates $u^{h}, v^{h}$ converge strongly, the discrete entropy inequality (4.12) gives a weak solution dissipating the mechanical energy.

\section{Existence of minimizers}

Henceforth, we consider stored-energy functions (3.10) of the form

$$
\begin{aligned}
\Phi\left(v_{1}, v_{2}, v_{3}\right) & =\bar{G}\left(v_{1}, v_{2}, v_{3}, 2 v_{3}, v_{1} v_{3}, v_{1} v_{2}, v_{1} v_{2} v_{3}\right) \\
& =\varphi\left(v_{1}\right)+\varphi\left(v_{2}\right)+\varphi\left(v_{3}\right)+g\left(v_{2} v_{3}\right)+g\left(v_{1} v_{3}\right)+g\left(v_{1} v_{2}\right)+h\left(v_{1} v_{2} v_{3}\right)
\end{aligned}
$$

Then, the function $G$ defined in (3.12) reads

$$
\begin{aligned}
G(\Xi ; \rho)= & \varphi\left(\xi_{1}\right)+\varphi\left(\xi_{2}^{1 / 3}\right)+\varphi\left(\xi_{3}^{1 / 3}\right) \\
& +g\left(\xi_{4} \rho^{1 / 3}\right)+g\left(\xi_{5} \rho^{1 / 3}\right)+g\left(\xi_{6} \rho^{1 / 3}\right)+h\left(\xi_{7} \rho^{2 / 3}\right)
\end{aligned}
$$

Now, define $\psi(x)=\varphi\left(x^{1 / 3}\right)$. Then, with $\Xi$ defined in (4.2), the above is expressed by

$$
G(\Xi)=\varphi\left(\beta \rho^{2 / 3}\right)+2 \psi(\alpha / \rho)+g\left(\gamma / \rho^{2 / 3}\right)+2 g\left(3 \gamma^{\prime} \rho^{1 / 3} / 2\right)+h\left(\alpha^{\prime}\right)
$$

We place the following assumptions on the functions $\varphi, \psi, g, h$ appearing above:

(A1) $\lim _{\delta \rightarrow 0+} h(\delta)=\lim _{\delta \rightarrow+\infty} h(\delta) / \delta=+\infty$;

(A2) $\varphi, \psi, g \in C^{2}(\mathbb{R})$ and $h \in C^{2}\left(\mathbb{R}_{+}\right)$satisfy

$$
\varphi, \psi, g, h, \varphi^{\prime \prime}, \psi^{\prime \prime}, g^{\prime \prime} \geqslant 0 \quad \text { and } \quad h^{\prime \prime}>0
$$

(A3) For $1<p, q<\infty$ and some constants $c_{1}, c_{2}>0$,

$$
\lim _{x \rightarrow \infty} \frac{\varphi(x)}{|x|^{3 p}}=\lim _{x \rightarrow \infty} \frac{\psi(x)}{|x|^{p}}=c_{1}, \lim _{x \rightarrow \infty} \frac{g(x)}{|x|^{q}}=c_{2}
$$

(A4) For $1<p, q<\infty$ as in (A3) and $C_{1}, C_{2}, C_{3}>0$,

$$
\limsup _{x \rightarrow \infty} \frac{\left|\varphi^{\prime}(x)\right|}{|x|^{3 p-1}} \leq C_{1} \limsup _{x \rightarrow \infty} \frac{\left|\psi^{\prime}(x)\right|}{|x|^{p-1}} \leq C_{2}, \limsup _{x \rightarrow \infty} \frac{\left|g^{\prime}(x)\right|}{|x|^{q}} \leq C_{3} ;
$$

In particular, $\mathrm{G}$ is convex. 
We define spaces of functions on the interval $\rho \in(0,1)$

$$
\begin{aligned}
X_{1} & =\left\{f(\rho) \in W^{1,1}(0,1): f / \rho \in L^{p}(0,1)\right\}, \\
X_{2} & =\left\{f(\rho) \in L_{l o c}^{1}(0,1): f \rho^{2 / 3} \in L^{3 p}(0,1)\right\}, \\
X_{3} & =\left\{f(\rho) \in W_{l o c}^{1,1}(0,1): f / \rho^{2 / 3} \in L^{q}, f^{\prime} \rho^{1 / 3} \in L^{q}(0,1)\right\}, \\
Y & =\left\{f(\rho) \in W_{l o c}^{1,1}(0,1): f \in L^{2}, f^{\prime} \rho^{2 / 3} \in L^{3 p}(0,1)\right\}, \\
\text { and } & \\
X & =X_{1} \otimes X_{2} \otimes X_{3} \otimes Y .
\end{aligned}
$$

We fix a parameter $\lambda>0$ and for the initial data $\left(\alpha_{0}, \beta_{0}, \gamma_{0}, v_{0}\right) \in X$ we require

$$
\left\{\begin{array}{l}
\alpha_{0}(1)=\lambda, \quad \alpha_{0} \geqslant 0, \alpha_{0}^{\prime}>0, \text { a.e. } \rho \in(0,1), \\
\int_{0}^{1} \frac{1}{2} v_{0}^{2}+G\left(\Xi^{0}\right) d \rho<\infty .
\end{array}\right.
$$

Consider the problem of minimizing the functional

$$
\begin{aligned}
I(\alpha, \beta, \gamma, v)= & \int_{0}^{1}\left\{\frac{1}{2}\left(v-v_{0}\right)^{2}+G(\Xi)\right\} d \rho \\
= & \int_{0}^{1}\left\{\frac{1}{2}\left(v-v_{0}\right)^{2}+\varphi\left(\beta \rho^{2 / 3}\right)+2 \psi(\alpha / \rho)\right. \\
& \left.+g\left(\gamma / \rho^{2 / 3}\right)+2 g\left(3 \gamma^{\prime} \rho^{1 / 3} / 2\right)+h\left(\alpha^{\prime}\right)\right\} d \rho
\end{aligned}
$$

over the admissible set

$$
\begin{aligned}
& \mathcal{A}_{\lambda}=\left\{(\alpha, \beta, \gamma, v) \in X: \alpha(0) \geqslant 0, \alpha(1)=\lambda, \alpha^{\prime}>0\right. \text { a.e. and } \\
& I(\alpha, \beta, \gamma, v)<\infty, \frac{\left(\beta-\beta_{0}\right)}{h}=3 v^{\prime}, \\
& \left.\frac{\left(\alpha-\alpha_{0}\right)}{h}=3 \alpha_{0}{ }^{2 / 3} v, \frac{\left(\gamma-\gamma_{0}\right)}{h}=2 \alpha_{0}{ }^{1 / 3} v\right\} .
\end{aligned}
$$

We note that $I$ is well-defined for $(\alpha, \beta, \gamma, v) \in X$ with $\alpha^{\prime}>0$ a.e. $\rho \in(0,1)$, though $I$ might be equal to $\infty$.

Lemma 5.1. The admissible set $\mathcal{A}_{\lambda}$ is nonempty.

Proof. Take $(\alpha, \beta, \gamma, v)=\left(\alpha_{0}, \beta_{0}, \gamma_{0}, 0\right) \in X$. Then (5.7) implies $\alpha(0) \geqslant 0, \alpha(1)=\lambda$, $\alpha^{\prime}>0$ a.e., and

$$
I(\alpha, \beta, \gamma, v)=\int_{0}^{1}\left\{\frac{1}{2} v_{0}^{2}+G\left(\Xi^{0}\right)\right\} d \rho<\infty .
$$

Moreover the following holds: $\left(\beta-\beta_{0}\right) / h=0=3 v^{\prime},\left(\alpha-\alpha_{0}\right) / h=0=3 \alpha_{0}{ }^{2 / 3} v$, and $(\gamma-$ $\left.\gamma_{0}\right) / h=0=2 \alpha_{0}{ }^{1 / 3} v$. Hence $(\alpha, \beta, \gamma, v) \in \mathcal{A}_{\lambda}$. 
LEMMA 5.2 (I-bounded sequences). Let $\left\{\left(\alpha_{n}, \beta_{n}, \gamma_{n}, v_{n}\right)\right\}_{n \in \mathbb{N}} \subset \mathcal{A}_{\lambda}$ and

$$
M=\sup _{n \in \mathbb{N}} I\left(\alpha_{n}, \beta_{n}, \gamma_{n}, v_{n}\right)<\infty .
$$

Then $\exists(\alpha, \beta, \gamma, v) \in X$ and a subsequence $\left\{\left(\alpha_{\mu}, \beta_{\mu}, \gamma_{\mu}, v_{\mu}\right)\right\}$ s.t.

$$
\begin{array}{clcc}
\alpha_{\mu} \rightarrow \alpha & \text { in } W^{1,1}, & \alpha_{\mu} / \rho \rightarrow \alpha / \rho & \text { in } L^{p}, \\
\gamma_{\mu} / \rho^{2 / 3} \rightarrow \gamma / \rho^{2 / 3} & \text { in } L^{q}, & \gamma_{\mu}^{\prime} \rho^{1 / 3} \rightarrow \gamma^{\prime} \rho^{1 / 3} & \text { in } L^{q}, \\
v_{\mu} \rightarrow v & \text { in } L^{2}, & v_{\mu}^{\prime} \rho^{2 / 3} \rightarrow v^{\prime} \rho^{2 / 3} & \text { in } L^{3 p}, \\
\beta_{\mu} \rho^{2 / 3} \rightarrow \beta \rho^{2 / 3} & \text { in } L^{3 p} . & &
\end{array}
$$

Proof. First, $\alpha_{n} \geqslant 0, \alpha_{n}^{\prime}>0$ a.e. and $\alpha_{n}(1)=\lambda$ imply that $\left|\alpha_{n}\right| \leqslant \lambda$. Second, from (5.10) it follows that $\int_{0}^{1} h\left(\alpha_{n}^{\prime}\right) d \rho<M, \forall n$. By the de la Vallée Poussin criterion there exists $\alpha \in W^{1,1}$ and a subsequence $\left\{\alpha_{s}\right\}$ such that $\alpha_{s} \rightarrow \alpha$ weakly in $W^{1,1}$.

By (A3) there exist constants $C_{1}, C_{2}$ s.t. $\varphi(x) \geqslant C_{1}|x|^{3 p}-C_{2}, \psi(x) \geqslant C_{1}|x|^{p}-C_{2}$, and $g(x) \geqslant C_{1}|x|^{q}-C_{2}$, and thus

$$
\begin{aligned}
M & \geqslant I\left(\alpha_{s}, \beta_{s}, \gamma_{s}, v_{s}\right) \\
& \geqslant \int_{0}^{1} \frac{1}{2}\left(v_{s}-v_{0}\right)^{2} d \rho+C_{1} \int_{0}^{1}\left|\beta_{s} \rho^{2 / 3}\right|^{3 p}+2\left|\alpha_{s} / \rho\right|^{p}+\left|\gamma_{s} / \rho^{2 / 3}\right|^{q}+\frac{3}{2}\left|\gamma_{s}^{\prime} \rho^{1 / 3}\right|^{q} d \rho-4 C_{2} .
\end{aligned}
$$

This implies, for $1<p, q<\infty$, that $\alpha / \rho \in L^{p}$ and there exist $\beta \in X_{2}, \gamma \in X_{3}, v \in L^{2}$, and a subsequence $\left\{\alpha_{\mu}, \beta_{\mu}, \gamma_{\mu}, v_{\mu}\right\}$ of $\left\{\alpha_{s}, \beta_{s}, \gamma_{s}, v_{s}\right\}$ such that $(5.11)_{2,3,4,5,6}$ hold.

Finally, as $\left(\alpha_{\mu}, \beta_{\mu}, \gamma_{\mu}, v_{\mu}\right) \in \mathrm{A}_{\lambda}$ we have $3 v_{\mu}^{\prime} \rho^{2 / 3}=\left(\beta_{\mu}-\beta_{0}\right) \rho^{2 / 3} / h$. Then by $(5.11)_{3}$ we get $3 v_{\mu}^{\prime} \rho^{2 / 3} \rightarrow\left(\beta-\beta_{0}\right) \rho^{2 / 3} / h$ in $L^{3 p}$. It follows from $(5.11)_{6}$ that for each $f \in C_{0}^{\infty}(0,1)$

$$
\begin{aligned}
\int_{0}^{1} v f^{\prime} d \rho & =\lim _{\mu \rightarrow \infty} \int_{0}^{1} v_{\mu} f^{\prime} d \rho \\
& =-\lim _{\mu \rightarrow \infty} \int_{0}^{1} v_{\mu}^{\prime} f d \rho=-\int_{0}^{1} \frac{1}{3 h}\left(\beta-\beta_{0}\right) f d \rho
\end{aligned}
$$

and hence $v^{\prime}=\left(\beta-\beta_{0}\right) / 3 h$. Therefore $v \in Y$ and $v_{\mu}^{\prime} \rho^{2 / 3} \rightarrow v^{\prime} \rho^{2 / 3}$.

THEOREM 5.3 (Lower semi-continuity). Let $\left\{\left(\alpha_{n}, \beta_{n}, \gamma_{n}, v_{n}\right)\right\}_{n \in \mathbb{N}} \subset \mathcal{A}_{\lambda}$, $(\alpha, \beta, \gamma, v) \in X$ satisfy (5.10) and (5.11). Then $(\alpha, \beta, \gamma, v) \in \mathcal{A}_{\lambda}$ and

$$
I(\alpha, \beta, \gamma, v) \leqslant \liminf _{n \rightarrow \infty} I\left(\alpha_{n}, \beta_{n}, \gamma_{n}, v_{n}\right)=s<\infty .
$$

Proof. $\quad$ By hypothesis $0 \leqslant I_{n}=I\left(\alpha_{n}, \beta_{n}, \gamma_{n}, v_{n}\right) \leqslant M, \forall n \in \mathbb{N}$ and thus $s<\infty$. Recall that $\alpha_{n} \rightarrow \alpha$ weakly in $W^{1,1}$ and (along a subsequence) uniformly on $C[0,1]$. Since $\alpha_{n}(1)=\lambda$ we obtain $\alpha(1)=\lambda$. Moreover,

$$
\lim _{n \rightarrow \infty} \int_{0}^{1} \alpha_{n}^{\prime} \chi_{\left\{\alpha^{\prime}<0\right\}} d \rho=\int_{0}^{1} \alpha^{\prime} \chi_{\left\{\alpha^{\prime}<0\right\}} d \rho .
$$

Since $\alpha_{n}^{\prime}>0$ a.e. we obtain $\int_{0}^{1} \alpha^{\prime} \chi_{\left\{\alpha^{\prime}<0\right\}} d \rho \geqslant 0$, and thus $m\left\{\alpha^{\prime}<0\right\}=0$. 
Now, denote $A=\left\{\rho \in(0,1): \alpha^{\prime}=0\right\}$ and show that $m(A)=0$. We will argue by contradiction. Assume that $m(A)=\varepsilon>0$. Then (5.11) implies

$$
\lim _{n \rightarrow \infty} \int_{0}^{1} \alpha_{n}^{\prime} \chi_{A} d \rho=\int_{0}^{1} \alpha^{\prime} \chi_{A} d \rho=0
$$

Then, as $\alpha_{n}^{\prime}>0$ a.e., $\lim _{n \rightarrow \infty} \int_{0}^{1}\left|\alpha_{n}^{\prime} \chi_{A}\right| d \rho=0$. Hence $\alpha_{n}^{\prime} \chi_{A} \rightarrow 0$ in $L^{1}$. We extract a subsequence $\left\{\alpha_{n_{k}}^{\prime}\right\}$ such that $\alpha_{n_{k}}^{\prime} \chi_{A} \rightarrow 0$ a.e. $\rho \in(0,1)$. Now, by Egoroff's theorem there exists a measurable set $B \subset A$ such that $m(B)>\varepsilon / 2$ and $\alpha_{n_{k}}^{\prime} \rightarrow 0$ uniformly on $B$. Next, observe that

$$
\int_{0}^{1} h\left(\alpha_{n_{k}}^{\prime}\right) d \rho \geq \int_{B} h\left(\alpha_{n_{k}}^{\prime}\right) d \rho \geq m(B)\left(\inf _{\rho \in B} h\left(\alpha_{n_{k}}^{\prime}\right)\right)=: m(B) \mu_{n_{k}}
$$

Since $\mu_{n_{k}} \rightarrow \infty$ this contradicts (5.10). We conclude that $m(A)=0$.

Next we prove $\alpha \geqslant 0$ a.e. $\rho \in(0,1)$. Again $(5.11)_{1}$ implies

$$
\lim _{n \rightarrow \infty} \int_{0}^{1} \alpha_{n} \chi_{\{\alpha<0\}} d \rho=\int_{0}^{1} \alpha \chi_{\{\alpha<0\}} d \rho \geq 0,
$$

and thus $m\{\alpha<0\}=0$. This concludes that $\alpha$ satisfies all restrictions of membership in $\mathcal{A}_{\lambda}$.

Next, by (A2) we get

$$
\begin{aligned}
\varphi\left(\beta_{n} \rho^{2 / 3}\right) & \geqslant \varphi\left(\beta \rho^{2 / 3}\right)+\varphi^{\prime}\left(\beta \rho^{2 / 3}\right)\left(\beta_{n}-\beta\right) \rho^{2 / 3}, \\
\psi\left(\alpha_{n} / \rho\right) & \geqslant \psi(\alpha / \rho)+\psi^{\prime}(\alpha / \rho)\left(\alpha_{n}-\alpha\right) / \rho, \\
g\left(\gamma_{n} / \rho^{2 / 3}\right) & \geqslant g\left(\gamma / \rho^{2 / 3}\right)+g^{\prime}\left(\gamma / \rho^{2 / 3}\right)\left(\gamma_{n}-\gamma\right) / \rho^{2 / 3}, \\
g\left(3 \gamma_{n}^{\prime} \rho^{1 / 3} / 2\right) & \geqslant g\left(3 \gamma^{\prime} \rho^{1 / 3} / 2\right)+g^{\prime}\left(3 \gamma^{\prime} \rho^{1 / 3} / 2\right)\left(\gamma_{n}^{\prime}-\gamma\right) 3 \rho^{1 / 3} / 2
\end{aligned}
$$

a.e. $\rho \in(0,1)$. As $(\alpha, \beta, \gamma, v),\left(\alpha_{n}, \beta_{n}, \gamma_{n}, v_{n}\right) \in X$, from (A3) it follows that the right hand side of each of the inequalities in (5.18) are integrable and

$$
\begin{aligned}
& \varphi^{\prime}\left(\beta \rho^{2 / 3}\right) \in L^{\frac{3 p}{3 p-1}}, \psi^{\prime}(\alpha / \rho) \in L^{\frac{p}{p-1}} \\
& \text { and } g^{\prime}\left(\gamma / \rho^{2 / 3}\right), g^{\prime}\left(3 \gamma_{n}^{\prime} \rho^{1 / 3} / 2\right) \in L^{\frac{q}{q-1}} .
\end{aligned}
$$

Take an arbitrary $0<\delta<1$ and set $A_{\delta}=\left\{\rho \in(0,1): \delta \leqslant \alpha^{\prime} \leqslant 1 / \delta\right\}$. Then by (A2)

$$
h\left(\alpha_{n}^{\prime}\right) \geqslant h\left(\alpha^{\prime}\right) \chi_{A_{\delta}}+h^{\prime}\left(\alpha^{\prime}\right)\left(\alpha_{n}^{\prime}-\alpha^{\prime}\right) \chi_{A_{\delta}} \text { a.e. } \rho \in(0,1) .
$$

Moreover, (A1) and (A2) together imply

$$
\begin{aligned}
0 & \leqslant h\left(\alpha^{\prime}\right) \chi_{A_{\delta}}+\left|h^{\prime}\left(\alpha^{\prime}\right)\right| \chi_{A_{\delta}} \\
& \leqslant 2 \max \left(h(\delta), h(1 / \delta),\left|h^{\prime}(\delta)\right|,\left|h^{\prime}(1 / \delta)\right|\right) .
\end{aligned}
$$

Hence

$$
h\left(\alpha^{\prime}\right) \chi_{A_{\delta}}, h^{\prime}\left(\alpha^{\prime}\right) \chi_{A_{\delta}} \in L^{\infty},
$$

and we conclude that the right hand side of (5.20) is integrable. 
Finally,

$$
\left(v_{n}-v_{0}\right)^{2} \geqslant\left(v-v_{0}\right)^{2}+2\left(v-v_{0}\right)\left(v_{n}-v\right) \text { a.e. } \rho \in(0,1),
$$

where right hand side is integrable as $v, v_{n}, v_{0} \in L^{2}$.

Following the discussion above, (5.18)-(5.22) imply

$$
\begin{aligned}
I_{n} \geqslant \int_{0}^{1}\{ & \frac{1}{2}\left(v-v_{0}\right)^{2}+\varphi\left(\beta \rho^{2 / 3}\right)+2 \psi(\alpha / \rho) \\
& \left.+g\left(\gamma / \rho^{2 / 3}\right)+2 g\left(3 \gamma^{\prime} \rho^{1 / 3} / 2\right)\right\} d \rho+\int_{0}^{1} h\left(\alpha^{\prime}\right) \chi_{A_{\delta}} d \rho \\
& +\int_{0}^{1}\left\{\left(v-v_{0}\right)\left(v_{n}-v\right)+\varphi^{\prime}\left(\beta \rho^{2 / 3}\right)\left(\beta_{n}-\beta\right) \rho^{2 / 3}\right. \\
& +2 \psi^{\prime}(\alpha / \rho)\left(\alpha_{n}-\alpha\right) / \rho+g^{\prime}\left(\gamma / \rho^{2 / 3}\right)\left(\gamma_{n}-\gamma\right) / \rho^{2 / 3} \\
& \left.+g^{\prime}\left(3 \gamma^{\prime} \rho^{1 / 3} / 2\right)\left(\gamma_{n}^{\prime}-\gamma\right) 3 \rho^{1 / 3}+h^{\prime}\left(\alpha^{\prime}\right) \chi_{A_{\delta}}\left(\alpha_{n}^{\prime}-\alpha^{\prime}\right)\right\} d \rho \\
=J+ & J_{\delta}+J_{n} .
\end{aligned}
$$

Then, letting $n \rightarrow \infty$, we obtain

$$
\infty>s=\liminf _{n \rightarrow \infty} I_{n} \geqslant J+J_{\delta}+\liminf _{n \rightarrow \infty} J_{n} .
$$

Now from $(5.11),(5.19),(5.21)$, and $v-v_{0} \in L^{2}$ it follows that $\lim _{n \rightarrow \infty} J_{n}=0$ and hence

$$
\infty>s=\liminf _{n \rightarrow \infty} I_{n} \geqslant J+\int_{0}^{1} h\left(\alpha^{\prime}\right) \chi_{A_{\delta}} d \rho .
$$

Now, as $\alpha^{\prime}>0$ a.e. $\rho \in(0,1)$ and $\alpha^{\prime} \in L^{1}$, the set $\left\{\alpha^{\prime}=0\right\} \bigcup\left\{\alpha^{\prime}=\infty\right\}$ is of measure zero, and hence

$$
\lim _{\delta \rightarrow 0+} h\left(\alpha^{\prime}\right) \chi_{A_{\delta}}=h\left(\alpha^{\prime}\right) \chi_{\left\{0<\alpha^{\prime}<\infty\right\}}=h\left(\alpha^{\prime}\right) \text { a.e. } \rho \in(0,1) .
$$

Finally, let $\delta \rightarrow 0+$. Then from (5.23), (5.24), and the Monotone Convergence Theorem it follows that

$$
\infty>s=\liminf _{n \rightarrow \infty} I_{n} \geqslant J+\int_{0}^{1} h\left(\alpha^{\prime}\right) d \rho=I(\alpha, \beta, \gamma, v)
$$

and hence (5.14) holds. Since $\left(\alpha_{n}, \beta_{n}, \gamma_{n}, v_{n}\right) \in \mathcal{A}_{\lambda}$, and the other constraints are linear, one easily checks that the limiting $(\alpha, \beta, \gamma, v) \in \mathcal{A}_{\lambda}$.

TheOREM 5.4 (Existence). There exists $(\alpha, \beta, \gamma, v) \in \mathcal{A}_{\lambda}$ satisfying

$$
I(\alpha, \beta, \gamma, v)=\inf _{\mathcal{A}_{\lambda}} I(\bar{\alpha}, \bar{\beta}, \bar{\gamma}, \bar{v}) .
$$

Proof. As $\mathcal{A}_{\lambda}$ is nonempty, we can set $s=\inf _{\mathcal{A}_{\lambda}} I(\bar{\alpha}, \bar{\beta}, \bar{\gamma}, \bar{v})$. Then by definition of $\mathcal{A}_{\lambda}$ we have $I(\bar{\alpha}, \bar{\beta}, \bar{\gamma}, \bar{v})<\infty$ for each $(\bar{\alpha}, \bar{\beta}, \bar{\gamma}, \bar{v}) \in \mathcal{A}_{\lambda}$. This implies that $s$ is finite.

Next, by definition of $s$ there exists $\left\{\left(\alpha_{n}, \beta_{n}, \gamma_{n}, v_{n}\right)\right\}_{n \in \mathbb{N}} \in \mathcal{A}_{\lambda}$ such that $\lim _{n \rightarrow \infty} I_{n}=s$ with $I_{n}=I\left(\alpha_{n}, \beta_{n}, \gamma_{n}, v_{n}\right)$. Then, as $\left\{I_{n}\right\}_{n \in \mathbb{N}}$ is bounded, Lemma 5.2 
and Theorem 5.3 imply that $\exists(\alpha, \beta, \gamma, v) \in \mathcal{A}_{\lambda}$ satisfying $I(\alpha, \beta, \gamma, v) \leqslant \liminf _{n \rightarrow \infty} I_{n}=$ $s$. In this case the definition of $s$ implies $I(\alpha, \beta, \gamma, v)=s$.

TheOrem 5.5 (Uniqueness). The minimizer $(\alpha, \beta, \gamma, v) \in \mathcal{A}_{\lambda}$ of I over $\mathcal{A}_{\lambda}$ is unique.

Proof. We will argue by contradiction. Assume $(\alpha, \beta, \gamma, v),(\bar{\alpha}, \bar{\beta}, \bar{\gamma}, \bar{v}) \in \mathcal{A}_{\lambda}$ are two distinct minimizers. Then we consider $\left(\frac{\alpha+\bar{\alpha}}{2}, \frac{\beta+\bar{\beta}}{2}, \frac{\gamma+\bar{\gamma}}{2}, \frac{v+\bar{v}}{2}\right)$ and notice that it also belongs to $\mathcal{A}_{\lambda}$.

Define $A=\left\{\rho \in(0,1): \alpha^{\prime} \neq \bar{\alpha}^{\prime}\right\}$. Then $m A>0$. Indeed, if $\alpha^{\prime}=\bar{\alpha}^{\prime}$ a.e., then $\alpha(1)=$ $\bar{\alpha}(1)=\lambda$ implies $\alpha=\bar{\alpha}$. In turn, this implies $v=\bar{v}^{\prime}, \beta=\bar{\beta}$, and $\gamma=\bar{\gamma}$, which contradicts to the assumption that $(\alpha, \beta, \gamma, v)$ and $(\bar{\alpha}, \bar{\beta}, \bar{\gamma}, \bar{v})$ are distinct.

Now, as $h^{\prime \prime}>0$, we have

$$
\frac{h\left(\alpha^{\prime}\right)+h\left(\bar{\alpha}^{\prime}\right)}{2}>h\left(\frac{\alpha^{\prime}+\bar{\alpha}^{\prime}}{2}\right), \rho \in A,
$$

and thus, as $m A$ is positive,

$$
\int_{0}^{1} \frac{h\left(\alpha^{\prime}\right)+h\left(\bar{\alpha}^{\prime}\right)}{2} d \rho>\int_{0}^{1} h\left(\frac{\alpha^{\prime}+\bar{\alpha}^{\prime}}{2}\right) d \rho .
$$

Let $s=\inf _{\mathcal{A}_{\lambda}} I(\tilde{\alpha}, \tilde{\beta}, \tilde{\gamma}, \tilde{v})$. Then by the inequality above and convexity of $\varphi, \psi$ and $g$ we obtain

$$
s=\frac{I(\alpha, \beta, \gamma, v)+I(\bar{\alpha}, \bar{\beta}, \bar{\gamma}, \bar{v})}{2}>I\left(\frac{\alpha+\bar{\alpha}}{2}, \frac{\beta+\bar{\beta}}{2}, \frac{\gamma+\bar{\gamma}}{2}, \frac{v+\bar{v}}{2}\right),
$$

which, since $\left(\frac{\alpha+\bar{\alpha}}{2}, \frac{\beta+\bar{\beta}}{2}, \frac{\gamma+\bar{\gamma}}{2}, \frac{v+\bar{v}}{2}\right) \in \mathcal{A}_{\lambda}$, contradicts the definition of $s$. Hence $(\alpha, \beta, \gamma, v)=(\bar{\alpha}, \bar{\beta}, \bar{\gamma}, \bar{v})$.

\section{Euler-Lagrange equations}

Next, we show that the minimizer of $I$ satisfies the system $(4.3)$ a.e. $\rho \in(0,1)$. To this end, in addition to (5.7), we assume that the initial iterate $\left(\alpha_{0}, \beta_{0}, \gamma_{0}, v_{0}\right)$ satisfies for each $\delta \in(0,1)$

$$
\alpha_{0}^{\prime} \in L^{3 p}(\delta, 1) \bigcap L^{q}(\delta, 1) .
$$

TheOREM 6.1 (Weak Form). Let $(\alpha, \beta, \gamma, v) \in \mathcal{A}_{\lambda}$ be the minimizer of I over $\mathcal{A}_{\lambda}$ and the initial iterate $\left(\alpha_{0}, \beta_{0}, \gamma_{0}, v_{0}\right)$ satisfy (5.7) and (6.1). Let also

$$
G_{1}(\rho)=G_{, i}(\Xi) \Omega_{, 1}^{i}\left(\Gamma^{0}\right)
$$

and

$$
G_{2}(\rho)=G_{, i}(\Xi)\left(\Omega_{, 2}^{i}\left(\Gamma^{0}\right)+\Omega_{, 3}^{i}\left(\Gamma^{0}\right)\right) .
$$

Then, for each $\delta \in(0,1)$,

$$
\rho^{2 / 3} G_{1}(\rho) \in W^{1,1}(\delta, 1), \quad \rho^{-1 / 3} G_{2}(\rho) \in L^{1}(\delta, 1),
$$

and for a.e. $\rho \in(0,1)$

$$
3 \rho^{2 / 3} G_{1}(\rho)=\int_{1}^{\rho}\left(s^{-1 / 3} G_{2}(s)+\frac{v(s)-v_{0}(s)}{h}\right) d s+\text { const. }
$$


Moreover, for each $\delta \in(0,1)$,

$$
\alpha^{\prime} \in L^{3 p}(\delta, 1) \bigcap L^{q}(\delta, 1) .
$$

Proof. Fix $k \in \mathbb{N}$ and define $S_{k}=\left\{\rho \in[1 / k, 1): 1 / k<\alpha^{\prime}<k\right\}$. Let $f \in L^{\infty}$ with $\int_{S_{k}} f d \rho=0$. We denote $\chi_{k}=\chi_{S_{k}}, l_{k}=\alpha_{0}(1 / k)$, and set

$$
\mu(\rho)=\int_{0}^{\rho} \chi_{k}(s) f(s) d s .
$$

Before proceeding further we make the following remark. Let $t \in \mathbb{R}$ and $F(x)=$ $x^{t}, x \in \mathbb{R}_{+}$. Take $\delta \in(0,1)$. Then, as $\alpha_{0} \in W^{1,1}, \alpha_{0} \geqslant 0$, and $\alpha_{0}^{\prime}>0$ a.e. $\rho \in(0,1)$ we must have $0<\alpha_{0}(\delta) \leqslant \alpha_{0} \leqslant \lambda$ for all $\rho \in(\delta, 1)$. Hence $\left|F^{\prime}\left(\alpha_{0}\right)\right| \leqslant t\left(\alpha_{0}(\delta)+\lambda\right)^{t-1}$ for all $\rho \in(\delta, 1)$. Therefore we conclude that for each $t \in \mathbb{R}$ and $\delta \in(0,1)$

$$
\alpha_{0}{ }^{t} \in W^{1,1}(\delta, 1) \text { with } \frac{d}{d \rho}\left(\alpha_{0}{ }^{t}\right)=t \alpha_{0}{ }^{t-1} \alpha_{0}^{\prime} .
$$
by

(i) Step 1. Definition of the variation. For $|\varepsilon|<\frac{1}{6 k\left(\|f\|_{\infty}+1\right)}$ we define $\left(\alpha_{\varepsilon}, \beta_{\varepsilon}, \gamma_{\varepsilon}, v_{\varepsilon}\right)$

$$
\begin{aligned}
& v_{\varepsilon}=v+\varepsilon \frac{\mu}{h \alpha_{0}^{2 / 3}}, \\
& \alpha_{\varepsilon}=\alpha_{0}+h\left(3 v_{\varepsilon} \alpha_{0}^{2 / 3}\right)=\alpha+3 \varepsilon \mu, \\
& \beta_{\varepsilon}=\beta_{0}+h\left(3 v_{\varepsilon}^{\prime}\right)=\beta+3 \varepsilon\left(\frac{\mu}{\alpha_{0}^{2 / 3}}\right)^{\prime}, \\
& \gamma_{\varepsilon}=\gamma_{0}+h\left(2 v_{\varepsilon} \alpha_{0}^{1 / 3}\right)=\gamma+2 \varepsilon \frac{\mu}{\alpha_{0}^{1 / 3}} .
\end{aligned}
$$

Due to $(6.7),\left(\alpha_{\varepsilon}, \beta_{\varepsilon}, \gamma_{\varepsilon}, v_{\varepsilon}\right)$ is well-defined. We next prove:

Lemma 6.2. The variation $\left(\alpha_{\varepsilon}, \beta_{\varepsilon}, \gamma_{\varepsilon}, v_{\varepsilon}\right) \in \mathcal{A}_{\lambda}$.

Proof. First, we notice that

$$
\left(\alpha_{\varepsilon}, \beta_{\varepsilon}, \gamma_{\varepsilon}, v_{\varepsilon}\right)=(\alpha, \beta, \gamma, v) \text { if } \rho \in(0,1 / k) .
$$

Then we check that

$$
\alpha_{\varepsilon}(1)=\alpha(1)+3 \varepsilon \int_{S_{k}} f(s) d s=\lambda .
$$

Next, we see that $\alpha_{\varepsilon}^{\prime}=\alpha^{\prime}+3 \varepsilon \chi_{k} f$ and therefore

$$
\begin{array}{r}
\alpha_{\varepsilon}^{\prime}=\alpha^{\prime}, \rho \notin S_{k}, \\
\frac{1}{2 k} \leqslant \alpha_{\varepsilon}^{\prime} \leqslant k+1, \rho \in S_{k} .
\end{array}
$$

This implies that $\alpha_{\varepsilon}>0$ a.e. $\rho \in(0,1)$ and hence (6.9) implies $\alpha_{\varepsilon} \geqslant 0$.

Now we make the following estimates. First, we see that

$$
\left|\mu^{\prime}\right|+\left|\frac{\mu}{\rho}\right|+\left|\frac{\mu}{\rho^{2 / 3} \alpha_{0}{ }^{1 / 3}}\right|+\left|\frac{\mu}{h \alpha_{0}^{2 / 3}}\right| \leqslant\|f\|_{\infty}\left(1+k+\frac{k^{2 / 3}}{l_{k}^{1 / 3}}+\frac{1}{h l_{k}^{2 / 3}}\right)
$$


and, for $j=1,2$,

$$
\left|\left(\frac{\mu}{\alpha_{0}^{j / 3}}\right)^{\prime}\right| \leqslant\|f\|_{\infty}\left(l_{k}^{-j / 3}+l_{k}^{-(1+j / 3)}\left|\alpha_{0}^{\prime}\right|\right) .
$$

Thus we conclude that there exists $C$ such that $\forall \rho \in(1 / k, 1)$

$$
\left|\alpha_{\varepsilon}^{\prime}-\alpha^{\prime}\right|+\left|\alpha_{\varepsilon} / \rho-\alpha / \rho\right|+\left|\gamma_{\varepsilon} / \rho^{2 / 3}-\gamma / \rho^{2 / 3}\right|+\left|v-v_{\varepsilon}\right| \leqslant \varepsilon C
$$

and

$$
\left|\beta_{\varepsilon} \rho^{2 / 3}-\beta \rho^{2 / 3}\right|+\left|\gamma_{\varepsilon}^{\prime} \rho^{1 / 3}-\gamma^{\prime} \rho^{1 / 3}\right| \leqslant \varepsilon C\left(1+\left|\alpha_{0}^{\prime}\right|\right) .
$$

As $(\alpha, \beta, \gamma, v) \in X$, the last two inequalities imply $\left(\alpha_{\varepsilon}, \beta_{\varepsilon}, \gamma_{\varepsilon}, v_{\varepsilon}\right) \in X$.

Further, by (A3), (6.11), and (6.12) we conclude that there exists $C$ such that for all $\rho \in(1 / k, 1)$

$$
\begin{aligned}
\psi\left(\alpha_{\varepsilon} / \rho\right) & \leqslant C\left(|\alpha / \rho|^{p}+1\right), \\
\varphi\left(\beta_{\varepsilon} \rho^{2 / 3}\right) & \leqslant C\left(\left|\beta \rho^{2 / 3}\right|^{3 p}+\left|\alpha_{0}^{\prime}\right|^{3 p}+1\right), \\
g\left(\gamma_{\varepsilon} / \rho^{2 / 3}\right) & \leqslant C\left(\left|\gamma / \rho^{2 / 3}\right|^{q}+1\right), \\
g\left(3 \gamma_{\varepsilon}^{\prime} \rho^{1 / 3} / 2\right) & \leqslant C\left(\left|\gamma^{\prime} \rho^{1 / 3}\right|^{q}+\left|\alpha_{0}^{\prime}\right|^{q}+1\right) .
\end{aligned}
$$

By (6.10) we also have

$$
\begin{aligned}
& h\left(\alpha_{\varepsilon}^{\prime}\right)=h\left(\alpha^{\prime}\right), \rho \notin S_{k}, \\
& h\left(\alpha_{\varepsilon}^{\prime}\right) \leqslant \max _{\frac{1}{2 k} \leqslant \delta \leqslant k}|h(\delta)|=M_{k}, \rho \in S_{k},
\end{aligned}
$$

and hence

$$
h\left(\alpha_{\varepsilon}^{\prime}\right) \leqslant h\left(\alpha^{\prime}\right)+M_{k}, \rho \in(0,1) .
$$

Now, similarly to (4.2), set

$$
\Xi_{\varepsilon}=\left(\beta_{\varepsilon} \rho^{2 / 3}, \frac{\alpha_{\varepsilon}}{\rho}, \frac{\alpha_{\varepsilon}}{\rho}, \frac{\gamma_{\varepsilon}}{\rho^{1 / 3}}, \frac{3 \gamma_{\varepsilon}^{\prime}}{2} \rho^{2 / 3}, \frac{3 \gamma_{\varepsilon}^{\prime}}{2} \rho^{2 / 3}, \alpha_{\varepsilon}^{\prime} \rho^{2 / 3}\right) .
$$

Then, by the discussion above, it follows that

$$
G\left(\Xi_{\varepsilon}\right)+\frac{\left(v_{\varepsilon}-v_{0}\right)^{2}}{2}=G(\Xi)+\frac{\left(v-v_{0}\right)^{2}}{2}, \rho \in(0,1 / k),
$$

and there exists $C$ such that for $\rho \in(1 / k, 1)$

$$
\begin{aligned}
G\left(\Xi_{\varepsilon}\right)+\frac{\left(v_{\varepsilon}-v_{0}\right)^{2}}{2} \leqslant C & \left(1+\left|\beta \rho^{2 / 3}\right|^{3 p}+\left|\alpha_{0}^{\prime}\right|^{3 p}+|\alpha / \rho|^{p}+\left|\gamma / \rho^{2 / 3}\right|^{q}\right. \\
& \left.+\left|\gamma^{\prime} \rho^{1 / 3}\right|^{q}+\left|\alpha_{0}^{\prime}\right|^{q}+|v|^{2}+\left|v_{0}\right|^{2}+h\left(\alpha^{\prime}\right)\right) .
\end{aligned}
$$

As $I(\alpha, \beta, \gamma, v)<\infty,(6.16)$ and (6.17) imply $I\left(\alpha_{\varepsilon}, \beta_{\varepsilon}, \gamma_{\varepsilon}, v_{\varepsilon}\right)<\infty$ and hence by construction and the above discussion we get $\left(\alpha_{\varepsilon}, \beta_{\varepsilon}, \gamma_{\varepsilon}, v_{\varepsilon}\right) \in \mathcal{A}_{\lambda}$. 
Step 2. The next objective is to validate the formal identity

$$
\left.\frac{d}{d \varepsilon} I\left(\alpha_{\varepsilon}, \beta_{\varepsilon}, \gamma_{\varepsilon}, v_{\varepsilon}\right)\right|_{\varepsilon=0}=\left.\int_{0}^{1} \frac{d}{d \varepsilon}\left(\frac{\left(v_{\varepsilon}-v_{0}\right)^{2}}{2}+G\left(\Xi_{\varepsilon}\right)\right)\right|_{\varepsilon=0} d \rho=0 .
$$

This will require several detailed estimations presented below.

At this point, let us make estimates of the following difference quotients on the interval $\rho \in(1 / k, 1)$. First, by $(6.11)$ we get

$$
\begin{aligned}
\frac{1}{\varepsilon}\left|\left(v_{\varepsilon}-v_{0}\right)^{2}-\left(v-v_{0}\right)^{2}\right| & =\frac{1}{\varepsilon}\left|v_{\varepsilon}-v\right|\left|v_{\varepsilon}+v-2 v_{0}\right| \\
& \leqslant C\left(|v|+\left|v_{0}\right|+1\right) .
\end{aligned}
$$

Further, by the Mean Value Theorem

$$
\frac{1}{\varepsilon}\left|\varphi\left(\beta_{\varepsilon} \rho^{2 / 3}\right)-\varphi\left(\beta \rho^{2 / 3}\right)\right|=\frac{1}{\varepsilon}\left|\varphi^{\prime}\left(\tau_{\varepsilon}\right)\right|\left|\beta_{\varepsilon} \rho^{2 / 3}-\beta \rho^{2 / 3}\right|,
$$

where $\min \left(\beta, \beta_{\varepsilon}\right) \rho^{2 / 3} \leqslant \tau_{\varepsilon} \leqslant \max \left(\beta, \beta_{\varepsilon}\right) \rho^{2 / 3}$. Hence from (6.12) it follows $\left|\tau_{\varepsilon}\right| \leqslant$ $\left|\beta \rho^{2 / 3}\right|+\varepsilon C\left(\left|\alpha_{0}^{\prime}\right|+1\right)$ and therefore (A4) implies

$$
\left|\varphi^{\prime}\left(\tau_{\varepsilon}\right)\right| \leqslant C\left(\left|\beta \rho^{2 / 3}\right|^{3 p-1}+\left|\alpha_{0}^{\prime}\right|^{3 p-1}+1\right) .
$$

Thus

$$
\begin{aligned}
& \frac{1}{\varepsilon}\left|\varphi\left(\beta_{\varepsilon} \rho^{2 / 3}\right)-\varphi\left(\beta \rho^{2 / 3}\right)\right| \\
\leqslant & C\left(\left|\beta \rho^{2 / 3}\right|^{3 p-1}+\left|\alpha_{0}^{\prime}\right|^{3 p-1}+1\right)\left(\left|\alpha_{0}^{\prime}\right|+1\right) .
\end{aligned}
$$

Similarly,

$$
\frac{1}{\varepsilon}\left|\psi\left(\alpha_{\varepsilon} / \rho\right)-\psi(\alpha / \rho)\right|=\frac{1}{\varepsilon}\left|\psi^{\prime}\left(\tau_{\varepsilon}\right)\right|\left|\alpha_{\varepsilon} / \rho-\alpha / \rho\right|,
$$

where $\min \left(\alpha_{\varepsilon}, \alpha\right) / \rho \leqslant \tau_{\varepsilon} \leqslant \max \left(\alpha_{\varepsilon}, \alpha\right) / \rho$. Hence $\left|\tau_{\varepsilon}\right| \leqslant|\alpha / \rho|+\varepsilon C$ and (A4) implies

$$
\left|\psi^{\prime}\left(\tau_{\varepsilon}\right)\right| \leqslant C\left((|\alpha / \rho|+1)^{p-1}+1\right)
$$

and hence

$$
\frac{1}{\varepsilon}\left|\psi\left(\alpha_{\varepsilon} / \rho\right)-\psi(\alpha / \rho)\right| \leqslant C\left((|\alpha / \rho|+1)^{p-1}+1\right) .
$$

Next,

$$
\frac{1}{\varepsilon}\left|g\left(\gamma_{\varepsilon} / \rho^{2 / 3}\right)-g\left(\gamma / \rho^{2 / 3}\right)\right|=\frac{1}{\varepsilon}\left|g^{\prime}\left(\tau_{\varepsilon}\right)\right|\left|\gamma_{\varepsilon} / \rho^{2 / 3}-\gamma / \rho^{2 / 3}\right|,
$$

where $\min \left(\gamma_{\varepsilon}, \gamma\right) / \rho^{2 / 3} \leqslant\left|\tau_{\varepsilon}\right| \leqslant \max \left(\gamma_{\varepsilon}, \gamma\right) / \rho^{2 / 3}$ and hence $\left|\tau_{\varepsilon}\right| \leqslant\left|\gamma / \rho^{2 / 3}\right|+\varepsilon C$. Then by (A4)

$$
\left|g^{\prime}\left(\tau_{\varepsilon}\right)\right| \leqslant C\left(\left(\left|\gamma / \rho^{2 / 3}\right|+1\right)^{q-1}+1\right)
$$

and hence

$$
\frac{1}{\varepsilon}\left|g\left(\gamma_{\varepsilon} / \rho^{2 / 3}\right)-g\left(\gamma / \rho^{2 / 3}\right)\right| \leqslant C\left(\left(\left|\gamma / \rho^{2 / 3}\right|+1\right)^{q-1}+1\right)
$$


Further,

$$
\frac{1}{\varepsilon}\left|g\left(3 \gamma_{\varepsilon}^{\prime} \rho^{1 / 3} / 2\right)-g\left(3 \gamma^{\prime} \rho^{1 / 3} / 2\right)\right|=\frac{3}{2 \varepsilon}\left|g^{\prime}\left(\tau_{\varepsilon}\right)\right|\left|\gamma_{\varepsilon}^{\prime} \rho^{1 / 3}-\gamma^{\prime} \rho^{1 / 3}\right|,
$$

where $\frac{3}{2} \min \left(\gamma_{\varepsilon}^{\prime}, \gamma^{\prime}\right) \rho^{1 / 3} \leqslant\left|\tau_{\varepsilon}\right| \leqslant \frac{3}{2} \max \left(\gamma_{\varepsilon}^{\prime}, \gamma^{\prime}\right) \rho^{1 / 3}$. Hence we must have $\left|\tau_{\varepsilon}\right| \leqslant$ $\frac{3}{2}\left(\left|\gamma^{\prime} \rho^{1 / 3}\right|+\varepsilon C\left(\left|\alpha_{0}^{\prime}\right|+1\right)\right)$. Then (A4) implies

$$
\left|g^{\prime}\left(\tau_{\varepsilon}\right)\right| \leqslant C\left(\left(\left|\gamma^{\prime} \rho^{1 / 3}\right|+\left|\alpha_{0}^{\prime}\right|+1\right)^{q-1}+1\right),
$$

and hence

$$
\begin{aligned}
& \frac{1}{\varepsilon}\left|g\left(3 \gamma_{\varepsilon}^{\prime} \rho^{1 / 3} / 2\right)-g\left(3 \gamma^{\prime} \rho^{1 / 3} / 2\right)\right| \\
\leqslant & C\left(\left(\left|\gamma^{\prime} \rho^{1 / 3}\right|+\left|\alpha_{0}^{\prime}\right|+1\right)^{q-1}+1\right)\left(\left|\alpha_{0}^{\prime}\right|+1\right) .
\end{aligned}
$$

Finally, if $\rho \notin S_{k}$ then $\frac{1}{\varepsilon}\left|h\left(\alpha_{\varepsilon}^{\prime}\right)-h\left(\alpha^{\prime}\right)\right|=0$, and if $\rho \in S_{k}$ we get

$$
\frac{1}{\varepsilon}\left|h\left(\alpha_{\varepsilon}^{\prime}\right)-h\left(\alpha^{\prime}\right)\right|=\frac{1}{\varepsilon}\left|h^{\prime}\left(\tau_{\varepsilon}\right)\right|\left|\alpha_{\varepsilon}^{\prime}-\alpha^{\prime}\right|,
$$

where $\min \left(\alpha_{\varepsilon}^{\prime}, \alpha^{\prime}\right) \leqslant \tau_{\varepsilon} \leqslant \max \left(\alpha_{\varepsilon}^{\prime}, \alpha\right)$. Then by $(6.10)_{2}$ we get $\frac{1}{2 k} \leqslant \tau_{\varepsilon} \leqslant k+1$ and hence

$$
\left|h^{\prime}\left(\tau_{\varepsilon}\right)\right| \leqslant \max _{\frac{1}{2 k} \leqslant \delta \leqslant k+1}\left|h^{\prime}(\delta)\right| .
$$

Thus by (6.11) we conclude that for $\rho \in(1 / k, 1)$

$$
\frac{1}{\varepsilon}\left|h\left(\alpha_{\varepsilon}^{\prime}\right)-h\left(\alpha^{\prime}\right)\right| \leqslant C \text {. }
$$

Thus (6.16),(6.19)-(6.24) and the assumptions on the initial iterate (5.7) and (6.1) imply that

$$
\frac{1}{\varepsilon}\left|G\left(\Xi_{\varepsilon}\right)+\frac{\left(v_{\varepsilon}-v_{0}\right)^{2}}{2}-G(\Xi)-\frac{\left(v-v_{0}\right)^{2}}{2}\right|
$$

is bounded on $(0,1)$ by an integrable function. Letting $\varepsilon \rightarrow 0$, and using the Dominated Convergence theorem, (A2) and the fact that $(\alpha, \beta, \gamma, v)$ is the minimizer, we obtain the identity (6.18).

Step 3. Conclusion of the computation. The last step is to compute the right hand side of (6.18). Note first that

$$
\begin{aligned}
& \frac{d \Xi_{\varepsilon}^{1}}{d \varepsilon}=\frac{d}{d \varepsilon} \beta_{\varepsilon} \rho^{2 / 3}=3\left(\frac{\mu}{\alpha_{0}^{2 / 3}}\right)^{\prime} \rho^{2 / 3}, \\
& \frac{d \Xi_{\varepsilon}^{2}}{d \varepsilon}=\frac{d \Xi_{\varepsilon}^{3}}{d \varepsilon}=\frac{d}{d \varepsilon}\left(\frac{\alpha_{\varepsilon}}{\rho}\right)=\frac{3 \mu}{\rho}, \\
& \frac{d \Xi_{\varepsilon}^{4}}{d \varepsilon}=\frac{d}{d \varepsilon}\left(\frac{\gamma \varepsilon}{\rho^{1 / 3}}\right)=\frac{2 \mu}{\alpha_{0}{ }^{1 / 3} \rho^{1 / 3}}, \\
& \frac{d \Xi_{\varepsilon}^{5}}{d \varepsilon}=\frac{d \Xi_{\varepsilon}^{6}}{d \varepsilon}=\frac{d}{d \varepsilon}\left(\frac{3}{2} \gamma_{\varepsilon}^{\prime} \rho^{2 / 3}\right)=3\left(\frac{\mu}{\alpha_{0}{ }^{1 / 3}}\right)^{\prime} \rho^{2 / 3}, \\
& \frac{d \Xi_{\varepsilon}^{7}}{d \varepsilon}=\frac{d}{d \varepsilon}\left(\alpha_{\varepsilon}^{\prime} \rho^{2 / 3}\right)=3 \mu^{\prime} \rho^{2 / 3},
\end{aligned}
$$


and

$$
\frac{d v_{\varepsilon}}{d \varepsilon}=\frac{\mu}{h \alpha_{0}^{2 / 3}} .
$$

Then the integrand in (6.18) is expressed by

$$
\left.\left(v-v_{0}\right) \frac{d v_{\varepsilon}}{d \varepsilon}\right|_{\varepsilon=0}+\left.G_{, i}(\Xi) \frac{d \Xi_{\varepsilon}^{i}}{d \varepsilon}\right|_{\varepsilon=0}=a \mu+b \mu^{\prime}
$$

where

$$
\begin{aligned}
a(\rho)= & -G_{, 1}(\Xi) \frac{2 \alpha_{0}^{\prime}}{\alpha_{0}{ }^{5 / 3}} \rho^{2 / 3}+G_{, 2}(\Xi) \frac{3}{\rho}+G_{, 3}(\Xi) \frac{3}{\rho} \\
& +G_{, 4}(\Xi) \frac{2}{\alpha_{0}^{1 / 3} \rho^{1 / 3}}-\left(G_{, 5}(\Xi)+G_{, 6}(\Xi)\right) \frac{\alpha_{0}^{\prime}}{\alpha_{0}{ }^{4 / 3}} \rho^{2 / 3}+\frac{\left(v-v_{0}\right)}{h \alpha_{0}^{2 / 3}}
\end{aligned}
$$

and

$$
b(\rho)=\frac{3 \rho^{2 / 3}}{\alpha_{0}^{2 / 3}}\left(G_{, 1}(\Xi)+G_{, 5}(\Xi) \alpha_{0}{ }^{1 / 3}+G_{, 6}(\Xi) \alpha_{0}{ }^{1 / 3}+G_{, 7}(\Xi) \alpha_{0}{ }^{2 / 3}\right) .
$$

Thus by (6.18) we have $\left(a \mu+b \mu^{\prime}\right) \in L^{1}$ and

$$
\int_{1 / k}^{1}\left(a \mu+b \mu^{\prime}\right) d \rho=0 .
$$

Now, we claim $a \in L^{1}(1 / k, 1)$. By (A3) and definition (5.2) of $G$ it follows that for $\rho \in(1 / k, 1)$

$$
\begin{gathered}
\left|G_{, 1}(\Xi) \frac{\alpha_{0}^{\prime}}{\alpha_{0}{ }^{5 / 3}} \rho^{2 / 3}\right| \leqslant\left|\frac{\varphi^{\prime}\left(\beta \rho^{2 / 3}\right) \alpha_{0}{ }^{\prime}}{l_{k}^{5 / 3}}\right| \leqslant C\left(\left|\beta \rho^{2 / 3}\right|^{3 p-1}+1\right)\left|\alpha_{0}^{\prime}\right|, \\
\frac{1}{\rho}\left|G_{, 2}(\Xi)+G_{, 3}(\Xi)\right| \leqslant 2 k\left|\psi^{\prime}(\alpha / \rho)\right| \leqslant C\left(|\alpha / \rho|^{p-1}+1\right),
\end{gathered}
$$

and

$$
\left|G_{, 4}(\Xi) \frac{1}{\alpha_{0}{ }^{1 / 3} \rho^{1 / 3}}\right| \leqslant \frac{k^{1 / 3}}{l_{k}^{1 / 3}}\left|g^{\prime}\left(\gamma / \rho^{2 / 3}\right)\right| \leqslant C\left(\left|\gamma / \rho^{2 / 3}\right|^{q-1}+1\right) .
$$

As the right hand sides of the inequalities above are integrable on $(1 / k, 1)$ we have $a \in$ $L^{1}(1 / k, 1)$ and this, in turn, implies $b \mu^{\prime} \in L^{1}(1 / k, 1)$. Now, we set $z(\rho)=\int_{1}^{\rho} a(s) d s$ for $\rho \in(1 / k, 1)$. Then $z$ is absolutely continuous and so is $\mu z$. As $\left.(\mu z)\right|_{\rho=1 / k}=\left.(\mu z)\right|_{\rho=1}=0$ we get

$$
0=\int_{1 / k}^{1}(\mu z)^{\prime} d \rho=\int_{1 / k}^{1}\left(\mu^{\prime} \int_{1}^{\rho} a(s) d s+\mu a\right) d \rho .
$$

Then (6.27) becomes

$$
\int_{S_{k}}\left(-\int_{1}^{\rho} a(s) d s+b\right) f d \rho=0 .
$$


By the properties of $f$ we obtain that for some constant $c_{k}$

$$
b-\int_{1}^{\rho} a(s) d s=c_{k} \text { a.e. } \rho \in S_{k} .
$$

As $k$ was arbitrary, the above equality is valid for all $k \in \mathbb{N}$. In this case $S_{k} \subset S_{k+1}$ implies that $c_{k}=c_{k+1}$. As $\bigcup_{k} S_{k}=\left\{\rho \in(0,1): 0<\alpha^{\prime}<\infty\right\}$ and $m\left((0,1) \backslash \bigcup_{k} S_{k}\right)=0$, we conclude

$$
b-\int_{1}^{\rho} a(s) d s=\text { const. a.e. } \rho \in(0,1) .
$$

Now, let us fix $\delta \in(0,1)$. By the above argument $a \in L^{1}(\delta, 1)$ and (6.29) implies $b \in W^{1,1}(\delta, 1)$ with the weak derivative $b^{\prime}=a$. Moreover, by $(6.7)$ we have $\alpha_{0}{ }^{2 / 3} \in$ $W^{1,1}(\delta, 1)$ and hence $b \alpha_{0}^{2 / 3} \in W^{1,1}(\delta, 1)$. At this point, we compute

$$
D \Omega\left(\Gamma^{0}\right)=\left[\begin{array}{ccccccc}
1 & 0 & 0 & 0 & \alpha_{0}{ }^{1 / 3} & \alpha_{0}{ }^{1 / 3} & \alpha_{0}{ }^{2 / 3} \\
0 & 3\left(\frac{\alpha_{0}}{\rho}\right)^{2 / 3} & 0 & \alpha_{0}{ }^{1 / 3} & 0 & \frac{\alpha_{0}^{\prime} \rho}{\alpha_{0}{ }^{2 / 3}} & \frac{\alpha_{0}^{\prime} \rho}{\alpha_{0}{ }^{1 / 3}} \\
0 & 0 & 3\left(\frac{\alpha_{0}}{\rho}\right)^{2 / 3} & \alpha_{0}{ }^{1 / 3} & \frac{\alpha_{0}^{\prime} \rho}{\alpha_{0}{ }^{2 / 3}} & 0 & \frac{\alpha_{0}^{\prime} \rho}{\alpha_{0} 1 / 3}
\end{array}\right]
$$

and notice that definitions (6.25) and (6.26) of $a$ and $b$ imply

$$
b \alpha_{0}{ }^{2 / 3}=3 \rho^{2 / 3} G_{, i}(\Xi) \Omega_{, 1}^{i}\left(\Gamma^{0}\right)=3 \rho^{2 / 3} G_{1}(\rho),
$$

while its weak derivative is expressed as

$$
\begin{aligned}
\frac{d}{d \rho} b \alpha_{0}{ }^{2 / 3} & =a \alpha_{0}{ }^{2 / 3}+b \frac{2 \alpha_{0}^{\prime}}{3 \alpha_{0}{ }^{1 / 3}} \\
& =\rho^{-1 / 3} G_{, i}(\Xi)\left(\Omega_{, 2}^{i}\left(\Gamma^{0}\right)+\Omega_{, 3}^{i}\left(\Gamma^{0}\right)\right)+\frac{v-v_{0}}{h} \\
& =\rho^{-1 / 3} G_{2}(\rho)+\frac{v-v_{0}}{h} .
\end{aligned}
$$

We conclude that, for $\delta \in(0,1)$,

$$
\rho^{2 / 3} G_{1}(\rho) \in W^{1,1}(\delta, 1), \quad \rho^{-1 / 3} G_{2}(\rho) \in L^{1}(\delta, 1),
$$

and for almost every $\rho \in(0,1)$

$$
3 \rho^{2 / 3} G_{1}(\rho)=\int_{1}^{\rho}\left(s^{-1 / 3} G_{2}(s)+\frac{v(s)-v_{0}(s)}{h}\right) d s+\text { const. }
$$

Finally, to prove (6.5), we compute

$$
\begin{aligned}
\left(\alpha-\alpha_{0}\right)^{\prime} & =h\left(3 \alpha_{0}{ }^{2 / 3} v\right)^{\prime}=h\left(\frac{2 \alpha_{0}^{\prime}}{\alpha_{0}{ }^{1 / 3}} v+3 \alpha_{0}{ }^{2 / 3} v^{\prime}\right) \\
& =\left(\alpha-\alpha_{0}\right) \frac{2 \alpha_{0}^{\prime}}{3 \alpha_{0}}+\left(\beta-\beta_{0}\right) \alpha_{0}{ }^{2 / 3},
\end{aligned}
$$

and hence

$$
\alpha^{\prime}=\frac{\alpha_{0}^{\prime}}{3}\left(1+\frac{2 \alpha}{\alpha_{0}}\right)+\left(\beta-\beta_{0}\right) \alpha_{0}^{2 / 3} .
$$


Similarly,

$$
\begin{aligned}
\left(\gamma-\gamma_{0}\right)^{\prime} & =h\left(2 \alpha_{0}{ }^{1 / 3} v\right)^{\prime}=\frac{2}{3}\left(\frac{\alpha-\alpha_{0}}{\alpha_{0}{ }^{1 / 3}}\right)^{\prime} \\
& =\frac{2}{3 \alpha_{0}{ }^{1 / 3}}\left(\alpha^{\prime}-\frac{\alpha_{0}^{\prime}}{3}\left(2+\frac{\alpha}{\alpha_{0}}\right)\right)
\end{aligned}
$$

and hence

$$
\alpha^{\prime}=\frac{\alpha_{0}^{\prime}}{3}\left(2+\frac{\alpha}{\alpha_{0}}\right)+\frac{3}{2}\left(\gamma^{\prime}-\gamma_{0}{ }^{\prime}\right) \alpha_{0}{ }^{1 / 3} .
$$

Now, take $\delta \in(0,1)$. Then from (6.32) and (6.33) it follows that for all $\rho \in(\delta, 1)$

$$
\left|\alpha^{\prime}\right| \leqslant \frac{\left|\alpha_{0}^{\prime}\right|}{3}\left(1+\frac{2 \lambda}{\alpha_{0}(\delta)}\right)+\left|\beta-\beta_{0}\right| \lambda^{2 / 3}
$$

and

$$
\left|\alpha^{\prime}\right| \leqslant \frac{\left|\alpha_{0}^{\prime}\right|}{3}\left(2+\frac{\lambda}{\alpha_{0}(\delta)}\right)+\frac{3}{2}\left|\gamma^{\prime}-\gamma_{0}{ }^{\prime}\right| \lambda^{1 / 3} .
$$

Since $\delta$ is arbitrary and $\beta-\beta_{0} \in L^{3 p}(\delta, 1), \gamma^{\prime}-\gamma_{0}^{\prime} \in L^{q}(\delta, 1)$, the assumption (6.1) and last two inequalities imply that for each $\delta \in(0,1)$

$$
\alpha^{\prime} \in L^{3 p}(\delta, 1) \bigcap L^{q}(\delta, 1) .
$$

This completes the proof.

\section{Regularity}

First, we claim that for each representative of the minimizer $(\alpha, \beta, \gamma, v) \in \mathcal{A}_{\lambda}$ in the theorem (6.1) we can alter $\alpha^{\prime}$ on a set of measure zero such that functions $G_{1}$ and $G_{2}$ defined in (6.2) and (6.3) satisfy

$$
3 \rho^{2 / 3} G_{1}(\rho)=\int_{1}^{\rho} s^{-1 / 3} G_{2}(s)+\frac{v(s)-v_{0}(s)}{h} d s+C_{0}, \text { for all } \rho \in(0,1] .
$$

Indeed, let us fix representatives $(\alpha, \beta, \gamma, v)$ and $\left(\alpha_{0}, \beta_{0}, \gamma_{0}, v_{0}\right)$. Define

$$
z(\rho)=\frac{1}{3 \rho^{2 / 3}} \int_{1}^{\rho} s^{-1 / 3} G_{2}(s)+\frac{v(s)-v_{0}(s)}{h} d s+C_{0}
$$

and let $A=\left\{\rho \in(0,1]: G_{1}(\rho) \neq z(\rho)\right\}$. Take any $\rho_{0} \in A$ and define

$$
y_{0}=\left.\left(z(\rho)-\varphi^{\prime}\left(\beta \rho^{2 / 3}\right)-2 g^{\prime}\left(3 \gamma^{\prime} \rho^{1 / 3}\right)\left(\alpha_{0} / \rho\right)^{1 / 3}\right)\right|_{\rho=\rho_{0}} .
$$

Then by (A1) and (A2) it follows that there exists a unique $x_{0}$ such that $h^{\prime}\left(x_{0}\right)=$ $y_{0}\left(\rho_{0} / \alpha_{0}\left(\rho_{0}\right)\right)^{2 / 3}$. Now, by definition of $G_{1}$ we have for all $\rho \in(0,1]$

$$
G_{1}(\rho)=\varphi^{\prime}\left(\beta \rho^{2 / 3}\right)+2 g^{\prime}\left(3 \gamma^{\prime} \rho^{1 / 3} / 2\right)\left(\alpha_{0} / \rho\right)^{1 / 3}+h^{\prime}\left(\alpha^{\prime}\right)\left(\alpha_{0} / \rho\right)^{2 / 3} .
$$

Thus assigning $\alpha^{\prime}\left(\rho_{0}\right)=x_{0}$ we get $G_{1}\left(\rho_{0}\right)=z\left(\rho_{0}\right)$. In the end, after altering this way $\alpha^{\prime}$ on the set $A$, we get that $G_{1}(\rho)=z(\rho)$ for all $\rho \in(0,1]$. Moreover by (6.4) we have $m A=0$ and this finishes the proof. 
The following regularity lemma requires a smoother initial iterate than before. In particular we prove:

Lemma 7.1 (Regularity). Let $(\alpha, \beta, \gamma, v) \in \mathcal{A}_{\lambda}$ be the minimizer of I over $\mathcal{A}_{\lambda}$. Assume that the initial iterate $\left(\alpha_{0}, \beta_{0}, \gamma_{0}, v_{0}\right)$ satisfies (5.7),

$$
\alpha_{0}, \gamma_{0} \in C^{1}(0,1] \text { and } \beta_{0} \in C(0,1] \text {. }
$$

Then

$$
\alpha, \gamma, v \in C^{1}(0,1] \text { and } \beta \in C(0,1]
$$

Proof. Clearly, we can pick a representative $(\alpha, \beta, \gamma, v)$ such that $\alpha, \gamma, v \in C(0,1]$. Proceeding as in (6.32) and (6.33), the constraints $\frac{\alpha-\alpha_{0}}{h}=3 \alpha_{0}{ }^{2 / 3} v, \frac{\gamma-\gamma_{0}}{h}=2 \alpha_{0}{ }^{1 / 3} v$, and $\frac{\beta-\beta_{0}}{h}=3 v^{\prime}$ imply for a.e. $\rho \in(0,1)$

$$
\beta \rho^{2 / 3}=\alpha^{\prime}\left(\rho / \alpha_{0}\right)^{2 / 3}+f_{1}(\rho)
$$

and

$$
\frac{3}{2} \gamma^{\prime} \rho^{1 / 3}=\alpha^{\prime}\left(\rho / \alpha_{0}\right)^{1 / 3}+f_{2}(\rho),
$$

where

$$
\begin{aligned}
& f_{1}(\rho)=\beta_{0} \rho^{2 / 3}-\frac{\alpha_{0}^{\prime} \rho^{2 / 3}}{3 \alpha_{0}^{2 / 3}}\left(1+\frac{2 \alpha}{\alpha_{0}}\right), \\
& f_{2}(\rho)=\frac{3}{2} \gamma_{0}^{\prime} \rho^{1 / 3}-\frac{\rho^{1 / 3}}{\alpha_{0}^{1 / 3}}\left(2+\frac{\alpha}{\alpha_{0}}\right) .
\end{aligned}
$$

We note that (7.3) implies that $f_{1}$ and $f_{2}$ are continuous on $(0,1]$ functions.

First, we alter $\beta$ and $\gamma^{\prime}$ so that equality in (7.5) and (7.6) holds for all $\rho \in(0,1)$. Hence by $(7.2)$ we have for all $\rho \in(0,1]$

$$
\begin{aligned}
G_{1}(\rho)= & \varphi^{\prime}\left(\alpha^{\prime}\left(\rho / \alpha_{0}\right)^{2 / 3}+f_{2}(\rho)\right) \\
& +2 g^{\prime}\left(\alpha^{\prime}\left(\rho / \alpha_{0}\right)^{1 / 3}+f_{1}(\rho)\right)\left(\alpha_{0} / \rho\right)^{1 / 3} \\
& +h^{\prime}\left(\alpha^{\prime}\right)\left(\alpha_{0} / \rho\right)^{2 / 3} .
\end{aligned}
$$

and this suggests to define $f: \mathbb{R}_{+} \times(0,1] \rightarrow \mathbb{R}$ by

$$
\begin{aligned}
f(x, \rho)= & \varphi^{\prime}\left(x\left(\rho / \alpha_{0}\right)^{2 / 3}+f_{2}(\rho)\right) \\
& +2 g^{\prime}\left(x\left(\rho / \alpha_{0}\right)^{1 / 3}+f_{1}(\rho)\right)\left(\alpha_{0} / \rho\right)^{1 / 3} \\
& +h^{\prime}(x)\left(\alpha_{0} / \rho\right)^{2 / 3} .
\end{aligned}
$$

Now, define $A=\left\{\rho \in(0,1]: G_{1}(\rho) \neq z(\rho)\right\}$. Clearly, $m A=0$ and note that from (7.7) it follows that

$$
G_{1}(\rho)=f\left(\alpha^{\prime}, \rho\right)=z(\rho), \rho \notin A .
$$


Take $\rho_{0} \in A$. Then, as $\rho_{0}>0$ and $\alpha_{0}\left(\rho_{0}\right)>0$, properties (A1)-(A3) imply that $f_{x}\left(x, \rho_{0}\right)>0$ for all $x \in \mathbb{R}_{+} ;$moreover, $\lim _{x \rightarrow 0+} f\left(x, \rho_{0}\right)=-\infty$ and $\lim _{x \rightarrow+\infty} f\left(x, \rho_{0}\right)=$ $+\infty$. Hence there exists a unique $x_{0} \in \mathbb{R}_{+}$such that $f\left(x_{0}, \rho_{0}\right)=z\left(\rho_{0}\right)$.

At this point we are ready to assign new values for $\alpha^{\prime}, \beta$, and $\gamma^{\prime}$. Define

$$
\alpha^{\prime}\left(\rho_{0}\right)=x_{0}, \beta\left(\rho_{0}\right)=\frac{x_{0}}{\alpha_{0}\left(\rho_{0}\right)^{2 / 3}}+\frac{f_{1}\left(\rho_{0}\right)}{\rho_{0}^{2 / 3}},
$$

and

$$
\gamma^{\prime}\left(\rho_{0}\right)=\frac{2}{3}\left(\frac{x_{0}}{\alpha_{0}\left(\rho_{0}\right)^{1 / 3}}+\frac{f_{2}\left(\rho_{0}\right)}{\rho_{0}^{1 / 3}}\right) .
$$

This implies that (7.5) and (7.6) hold at $\rho=\rho_{0}$, and hence by (7.2)

$$
G_{1}\left(\rho_{0}\right)=f\left(x_{0}, \rho_{0}\right)=f\left(\alpha^{\prime}\left(\rho_{0}\right), \rho_{0}\right)=z\left(\rho_{0}\right) .
$$

As $\rho_{0} \in A$ was arbitrary (7.9) and (7.10) imply

$$
G_{1}(\rho)=f\left(\alpha^{\prime}, \rho\right)=z(\rho), \rho \in(0,1] .
$$

Hence $G_{1}$ is continuous on $(0,1]$ and therefore $\alpha^{\prime}>0$ for all $\rho \in(0,1]$.

Now, let us assume $\rho_{k} \rightarrow \rho_{0}$ and $\alpha^{\prime}\left(\rho_{k}\right) \rightarrow l \in[0, \infty]$ with $\rho_{k}, \rho_{0} \in(0,1], k \in \mathbb{N}$. First, we claim that $l \in(0, \infty)$. Indeed, assume that $l=0$ or $l=+\infty$. Then by continuity of $\alpha_{0}$ we have $\alpha_{0}\left(\rho_{k}\right) \rightarrow \alpha_{0}\left(\rho_{0}\right)>0$ and hence properties (A1)-(A3), together with continuity of $f_{1}$ and $f_{2}$, imply $\lim _{k \rightarrow \infty} f\left(\alpha^{\prime}\left(\rho_{k}\right), \rho_{k}\right)=\mp \infty$ respectively. Thus by continuity of $G_{1}$ and (7.11) we have

$$
G_{1}\left(\rho_{0}\right)=\lim _{k \rightarrow \infty} G_{1}\left(\rho_{k}\right)=\lim _{k \rightarrow \infty} f\left(\alpha^{\prime}\left(\rho_{k}\right), \rho_{k}\right)=\mp \infty,
$$

which is a contradiction. Therefore we assume $l \in(0, \infty)$. As $f_{1}, f_{2}$ are continuous on $(0,1]$, we must have $\lim _{k \rightarrow \infty} f\left(\alpha^{\prime}\left(\rho_{k}\right), \rho_{k}\right)=f\left(l, \rho_{0}\right)$, and therefore by $(7.11)$ we get

$$
\begin{aligned}
f\left(\alpha^{\prime}\left(\rho_{0}\right), \rho_{0}\right)=G_{1}\left(\rho_{0}\right) & =\lim _{k \rightarrow \infty} G_{1}\left(\rho_{k}\right) \\
& =\lim _{k \rightarrow \infty} f\left(\alpha^{\prime}\left(\rho_{k}\right), \rho_{k}\right)=f\left(l, \rho_{0}\right) .
\end{aligned}
$$

By the strict monotonicity of $f\left(\cdot, \rho_{0}\right)$ we get $\alpha_{0}\left(\rho_{0}\right)=l$ and conclude that $\alpha^{\prime}$ is continuous on $(0,1]$.

Finally, from the discussion above it follows that equalities (7.5) and (7.6) hold for all $\rho \in(0,1]$. The continuity of $f_{1}, f_{2}$, and $\alpha^{\prime}$ imply $\beta, \gamma^{\prime} \in C(0,1]$. Moreover, as $\frac{\alpha-\alpha_{0}}{h}=3 \alpha_{0}^{2 / 3} v$ for all $\rho \in(0,1]$, we obtain $v \in C^{1}(0,1]$. This finishes the proof.

\section{REFERENCES}

[1] J.M. Ball, Convexity conditions and existence theorems in nonlinear elasticity, Arch. Rational Mech. Anal., 63, 337-403, 1977.

[2] J.M. Ball, J.C. Currie, and P.J. Olver, Null Lagrangians, weak continuity and variational problems of arbitrary order, J. Funct. Anal., 41, 135-174, 1981.

[3] J.M. Ball, Discontinuous equilibrium solutions and cavitation in nonlinear elasticity, Phil. Trans. of the Royal Society of London. Series A, Math. Phys. Sci., 306, 557-611, 1982. 
[4] Y. Brenier, Hydrodynamic structure of the augmented Born-Infeld equations, Arch. Rational Mech. Anal., 172, 65-91, 2004.

[5] P.G. Ciarlet, Mathematical Elasticity, North Holland, 1993.

[6] C.M. Dafermos, Hyperbolic Conservation Laws in Continuum Physics, Third edition, Grundlehren der Mathematischen Wissenschaften, Springer-Verlag, Berlin, 2010.

[7] S. Demoulini, D.M.A. Stuart, and A.E. Tzavaras, Construction of entropy solutions for one dimensional elastodynamics via time discretization, Ann. Inst. H. Poincaré Anal. Non Linéaire, 17, 711-731, 2000.

[8] S. Demoulini, D.M.A. Stuart, and A.E.Tzavaras, A variational approximation scheme for three dimensional elastodynamics with polyconvex energy, Arch. Rational Mech. Anal., 157, 325$344,2001$.

[9] M.E. Gurtin, Topics in Finite Elastisity, CBMS-NSF Regional Conference Series in Applied Mathematics, 35. Society for Industrial and Applied Mathematics (SIAM), Philadelphia, PA, 1981.

[10] C. Lattanzio and A.E. Tzavaras, Structural properties of stress relaxation and convergence from viscoelasticity to polyconvex elastodynamics, Arch. Rational Mech. Anal., 180, 449492, 2006.

[11] T. Qin, Symmetrizing nonlinear elastodynamic system, J. Elasticity, 50, 245-252, 1998.

[12] D. Serre, Hyperbolicity of the nonlinear models of Maxwell's equations, Arch. Rational Mech. Anal., 172, 309-331, 2004.

[13] D. Serre, Non-linear electromagnetism and special relativity, Discr. Cont. Dynam. Systems, 23, 435-453, 2009.

[14] C. Truesdell and W. Noll, The Non-linear Field Theories of Mechanics, Handbuch der Physik, III, Third edition, (S.Flügge), Berlin: Springer, 1965. 